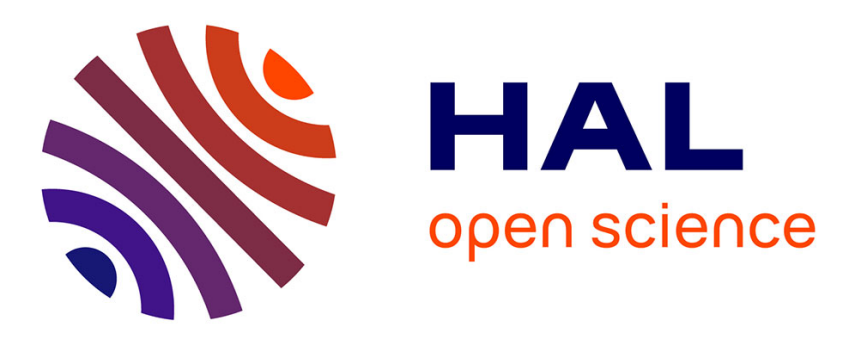

\title{
Residual-based a posteriori estimators for the A/phi magnetodynamic harmonic formulation of the Maxwell system
}

Emmanuel Creusé, Serge Nicaise, Zuqi Tang, Yvonnick Le Menach, Nicolas Nemitz, Francis Piriou

\section{To cite this version:}

Emmanuel Creusé, Serge Nicaise, Zuqi Tang, Yvonnick Le Menach, Nicolas Nemitz, et al.. Residual-based a posteriori estimators for the A/phi magnetodynamic harmonic formulation of the Maxwell system. Mathematical Models and Methods in Applied Sciences, 2012, 22 (5), pp.30. 10.1142/S021820251150028X . hal-00562073

\section{HAL Id: hal-00562073 https://hal.science/hal-00562073}

Submitted on 2 Feb 2011

HAL is a multi-disciplinary open access archive for the deposit and dissemination of scientific research documents, whether they are published or not. The documents may come from teaching and research institutions in France or abroad, or from public or private research centers.
L'archive ouverte pluridisciplinaire HAL, est destinée au dépôt et à la diffusion de documents scientifiques de niveau recherche, publiés ou non, émanant des établissements d'enseignement et de recherche français ou étrangers, des laboratoires publics ou privés. 


\title{
Residual-based a posteriori estimators for the $\mathbf{A}-\varphi$ magnetodynamic harmonic formulation of the Maxwell system
}

\author{
Emmanuel Creusé* \\ Serge Nicaise ${ }^{\dagger}$ \\ Zuqi Tang $\ddagger$ \\ Yvonnick Le Menach ${ }^{\S}$ \\ Nicolas Nemitz \\ Francis Pirioull
}

January 28, 2011

\begin{abstract}
This paper is devoted to the derivation of an a posteriori residual-based error estimator for the $\mathbf{A}-\varphi$ magnetodynamic harmonic formulation of the Maxwell system. The weak continuous and discrete formulations are established, and the wellposedness of both of them is addressed. Some useful analytical tools are derived. Among them, an ad-hoc Helmholtz decomposition is proven, which allows to pertinently split the error. Consequently, an a posteriori error estimator is obtained, which is proven to be reliable and locally efficient. Finally, numerical tests confirm the theoretical results.
\end{abstract}

Key Words : Maxwell equations, potential formulations, a posteriori estimators, finite element method.

AMS (MOS) subject classification 35Q61; 65N30; 65N15; 65N50.

${ }^{*}$ LPP UMR 8524 and INRIA Lille Nord Europe, Université de Lille 1, Cité Scientifique, 59655 Villeneuve d'Ascq Cedex, France, emmanuel.creuse@math.univ-lille1.fr.

${ }^{\dagger}$ LAMAV, FR CNRS 2956, Université de Valenciennes et du Hainaut Cambrésis, Institut des Sciences et Techniques de Valenciennes, F-59313 - Valenciennes Cedex 9, France.

${ }^{\ddagger}$ L2EP, Université de Lille 1, Cité Scientifique, 59655 Villeneuve d’Ascq Cedex,France.

${ }^{\S}$ L2EP, Université de Lille 1, Cité Scientifique, 59655 Villeneuve d'Ascq Cedex, France.

ฯTHEMIS, EDF R\&D, 1 avenue du Général de Gaulle, BP408, 92141 Clamart Cedex.

"L2EP, Université de Lille 1, Cité Scientifique, 59655 Villeneuve d'Ascq Cedex, France. 


\section{Introduction}

Let us consider the electromagnetic fields, modeled by the well-known Maxwell system :

$$
\begin{gathered}
\operatorname{rot} \mathbf{E}=-\frac{\partial \mathbf{B}}{\partial t}, \\
\operatorname{rot} \mathbf{H}=\frac{\partial \mathbf{D}}{\partial t}+\mathbf{J}
\end{gathered}
$$

where $\mathbf{E}$ is the electrical field, $\mathbf{H}$ the magnetic field, $\mathbf{B}$ the magnetic flux density, $\mathbf{J}$ the current flux density (or eddy current) and $\mathbf{D}$ the displacement flux density. Equation (1) is classically called Maxwell-Faraday equation and equation (2) Maxwell-Ampère one. In the low frequency regime, the quasistatic approximation can be applied, which consists in neglecting the temporal variation of the displacement flux density with respect to the current density [21], such that the propagation phenomena are not taken into account. Consequently, equation (2) becomes :

$$
\operatorname{rot} \mathbf{H}=\mathbf{J}
$$

Here, the current density $\mathbf{J}$ can be decomposed in two terms such that $\mathbf{J}=\mathbf{J}_{s}+\mathbf{J}_{e}$. $\mathbf{J}_{s}$ is a known distribution current density generally generated by a coil. $\mathbf{J}_{e}$ represents the eddy current. Both equations (1) and (3) are linked by the material constitutive laws :

$$
\begin{aligned}
& \mathbf{B}=\mu \mathbf{H}, \\
& \mathbf{J}_{\mathbf{e}}=\sigma \mathbf{E},
\end{aligned}
$$

where $\mu$ stands for the magnetic permeability and $\sigma$ for the electrical conductivity of the material. Figure 1 displays the domains configuration we are interested in. Let us consider an open connected domain $\Omega \subset \mathbb{R}^{3}$, with a lipschitz boundary $\Gamma=\partial \Omega$. We define an open connected conductor domain $\Omega_{c} \subset \Omega$ and we note $B=\partial \Omega_{c}$ its boundary which is supposed to be lipschitz and such that $B \cap \Gamma=\emptyset$. In $\Omega_{c}$, the electrical conductivity $\sigma$ is not equal to zero so that eddy currents can be created. Finally we define $\Omega_{e}=\Omega \backslash \overline{\Omega_{c}}$ as the part of $\Omega$ where the electrical conductivity $\sigma$ is equal to zero. Boundary conditions associated with the system (1)-(3) are B.n $=0$ on $\Gamma$ and $\mathbf{J}_{\mathbf{e}} \cdot \mathbf{n}=0$ on $B$. In the conductor domain $\Omega_{c}$, it is possible to solve the electromagnetic equations by only considering the electrical field, which leads to the classical $\mathbf{E}$ formulation :

$$
\operatorname{rot} \frac{1}{\mu} \operatorname{rot} \mathbf{E}+\sigma \frac{\partial \mathbf{E}}{\partial t}=0 .
$$

The same approach can be carried out with the magnetic field $\mathbf{H}$. In this case, we obtain the classical $\mathbf{H}$ formulation :

$$
\operatorname{rot} \frac{1}{\sigma} \operatorname{rot} \mathbf{H}+\mu \frac{\partial \mathbf{H}}{\partial t}=0
$$




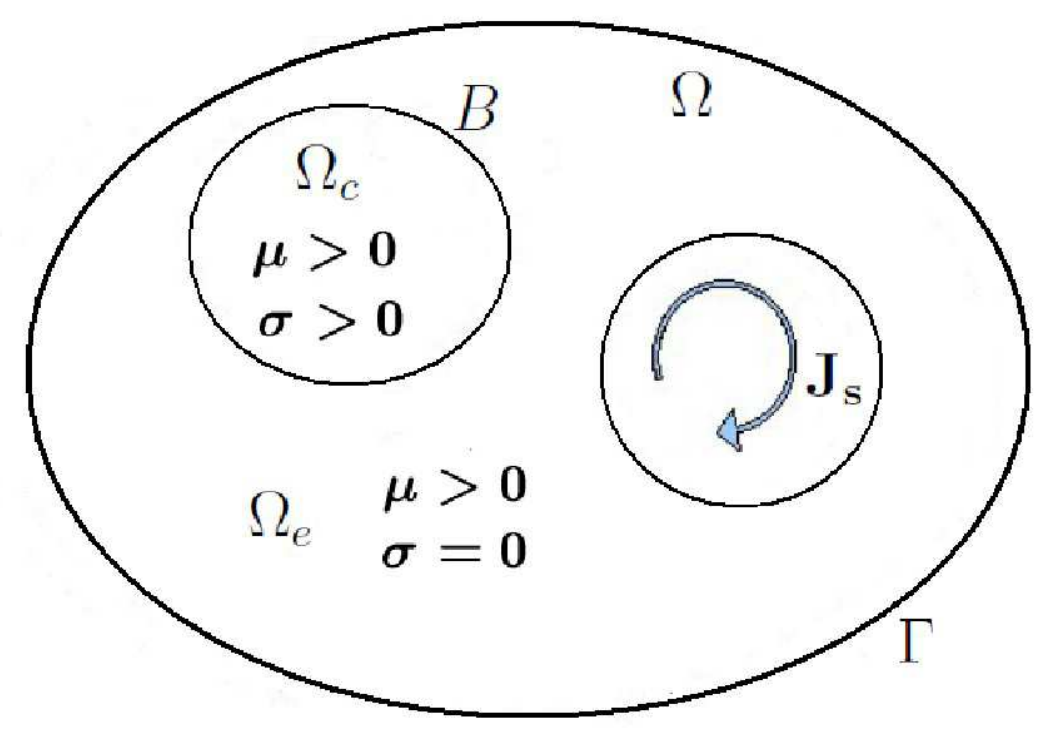

Figure 1: Domains configuration.

Unfortunately, these two formulations can only be considered in the conductor domain $\Omega_{c}$. Indeed, the electrical conductivity $\sigma$ and the eddy current exist only in $\Omega_{c}$. Then these formulations are banished if $\Omega_{e}$ exists. Consequently, in order to solve a problem with the quasistatic approximation, a formulation which is able to take into account the eddy current in $\Omega_{c}$ and which verifies in $\Omega_{e}$ Maxwell's equations reduced to $\operatorname{rot} \mathbf{H}=\mathbf{J}_{s}$ and $\operatorname{div} \mathbf{B}=0$ must be developed. That can be obtained by using the potentials formulations often used for electromagnetic problems [14]. From the property of the magnetic flux density B, a magnetic vector potential $\mathbf{A}$ can be introduced such that:

$$
\mathbf{B}=\operatorname{rot} \mathbf{A}
$$

with the boundary condition $\mathbf{A} \times \mathbf{n}=0$ on $\Gamma$ allowing to guarantee B.n $=0$ on $\Gamma$. Like $\mathbf{B}$, the vector potential $\mathbf{A}$ exists on the whole domain $\Omega$. To ensure the uniqueness of the solution, it is then necessary to impose a gauge condition. The most popular one (the Coulomb one) is to impose $\operatorname{div} \mathbf{A}=0$. Moreover, from equations (1) and (6), we get $\operatorname{rot}\left(\mathbf{E}+\frac{\partial \mathbf{A}}{\partial t}\right)=0$ and an electrical scalar potential $\varphi$ can be introduced so that the electrical field takes the form:

$$
\mathbf{E}=-\frac{\partial \mathbf{A}}{\partial t}-\nabla \varphi
$$

As explained before, due to the quasistatic approximation, $\mathbf{E}$ exists only in $\Omega_{c}$ and then the scalar potential $\varphi$ also only makes sense in $\Omega_{c}$. As for the vector potential, it must be gauged. To obtain uniqueness, the averaged value of the potentiel $\varphi$ on $\Omega_{c}$ can be taken equal to zero. From (4),(5),(6) and (7), equation (3) leads to the so-called $\mathbf{A}-\varphi$ formulation :

$$
\operatorname{rot}\left(\frac{1}{\mu} \operatorname{rot} \mathbf{A}\right)+\sigma\left(\frac{\partial \mathbf{A}}{\partial t}+\nabla \varphi\right)=\mathbf{J}_{s}
$$


The great interest of this formulation relies in its effectivity in both domain $\Omega_{c}$ and $\Omega_{e}$. Indeed, in $\Omega_{e}$ where $\sigma$ is null the second term vanishes and the $\mathbf{A}-\varphi$ formulation becomes the classical $\mathbf{A}$ formulation used in the magnetostatic case. Moreover, the conservation of the normal component $\mathbf{B}$ is naturally ensured on $B$. As the conductivity is null outside the conductor, the boundary conditions on the eddy current is also ensured.

In this paper, we are interested in deriving a reliable and efficient a posteriori residual error estimator for the finite element resolution of the $\mathbf{A}-\varphi$ formulation of the Maxwell equations given by (8), in its harmonic formulation (see (10)-(11) below for the corresponding weak formulation).

Isotropic a posteriori error estimators for standard elliptic boundary value problems are currently well understood $[1,2,17,24]$. The analysis of residual a posteriori error estimators for the edge elements, in the context of the E-formulation of Maxwell equations, was successivelly initiated in $[3,15]$ in the context of a "smooth" Helmholtz decomposition, with specific assumptions on the coefficients arising in the equations or on the domain regularity. Then, it was generalized to piecewise constant coefficients in [19] or to more general domains in [22], and the question of robustness with respect to the equation parameters was addressed in [8]. Among the now large bibliography devoted to a posteriori error analysis for $\mathbf{E}$-formulations, we can also quote [5] which introduces an adaptive edge element method for the static Maxwell equations. Some recently proposed estimators are based on reconstruction [18] or equilibrated strategies in order to derive asymptotically exactness properties [4]. Concerning the time-harmonic formulation in $\mathbf{E}$, an a posteriori residual based error estimator was recently proposed in [6], and an implicit a posteriori error estimator also derived in [12].

The originality of our work is twofold. First, we deal with a coupled formulation between the scalar potential $\varphi$ and the vectorial one $\mathbf{A}$, having moreover in mind that the supports of these two unknowns are not the same. Second, as it has already been mentioned above, each of these two potentials has to be gauged to ensure uniqueness of the solution (see $[13,20]$ for practical issues), leading to the fact that the approximation space for the vectorial potential is not included in the continuous one, and leading naturally to non conforming approximations.

The content of the paper is as follows. Section 2 establishes the weak formulation of the continuous and discrete problems, and proves the well-posedness of both of them. Section 3 is devoted to several analytical tools needed in the following. In particular, we derive an ad-hoc Helmholtz decomposition of the error, which has to take into account that the scalar potential is not defined outside of the conductor domain, and that an extension of it has to be properly defined. More usual tools are also recalled, such as suitable interpolation operators or the so-called "bubble functions". The reliability as well as the efficiency of the derived estimator are established in section 4. Finally, section 5 is devoted to numerical tests. The first is an analytical one for which the exact solution is known, allowing to 
confirm our theoretical analysis. The second one is an industrial test, which shows that the estimator is clearly relevant in real configurations.

\section{Weak formulation and discretization of the problem}

\subsection{Weak formulation}

Following (8) and assuming that $\operatorname{div} \mathbf{J}_{s}=0$, the $\mathbf{A}-\varphi$ formulation of the magnetodynamic problem can be written :

$$
\left\{\begin{aligned}
\operatorname{rot}\left(\frac{1}{\mu} \operatorname{rot} \mathbf{A}\right)+\sigma\left(\frac{\partial \mathbf{A}}{\partial t}+\nabla \varphi\right)=\mathbf{J}_{s} & \text { in } \Omega \\
\operatorname{div}\left(\sigma\left(\frac{\partial \mathbf{A}}{\partial t}+\nabla \varphi\right)\right)=0 & \text { in } \Omega_{c} .
\end{aligned}\right.
$$

We suppose that $\mu \in L^{\infty}(\Omega)$ and that there exists $\mu_{0} \in \mathbb{R}_{+}^{*}$ such that $\mu>\mu_{0}$ on $\Omega$. We also assume that, $\sigma \in L^{\infty}(\Omega), \sigma_{\mid \Omega_{e}} \equiv 0$, and that there exists $\sigma_{0} \in \mathbb{R}_{+}^{*}$ such that $\sigma>\sigma_{0}$ on $\Omega_{c}$. At last, we recall the Gauge conditions. Like mentioned in section 1 , we choose the Coulomb one $\operatorname{div} \mathbf{A}=0$ in $\Omega$, and we ask for the averaged value of $\varphi$ on $\Omega_{c}$ to be equal to zero. Now, the system (9) is considered in its harmonic formulation, namely we assume that $\mathbf{A}=\tilde{\mathbf{A}}(x) e^{j \omega t}$, with the notation $j^{2}=-1$ and the frequency $\omega$ is a fixed positive real number. Taking the associated boundary conditions into account, and writing $\mathbf{A}$ instead of $\tilde{\mathbf{A}}$, the variational formulation of (9) is now given by : Find $(\mathbf{A}, \varphi) \in V$ such that for all $\left(\mathbf{A}^{\prime}, \varphi^{\prime}\right) \in V$ we have :

$$
\begin{aligned}
\int_{\Omega} \frac{1}{\mu} \operatorname{rot} \mathbf{A} \cdot \operatorname{rot} \overline{\mathbf{A}^{\prime}}+\int_{\Omega_{c}} \sigma(j \omega \mathbf{A}+\nabla \varphi) \overline{\mathbf{A}^{\prime}} & =\int_{\Omega} \mathbf{J}_{s} \cdot \overline{\mathbf{A}^{\prime}} \text { in } \Omega, \\
\int_{\Omega_{c}} \sigma(j \omega \mathbf{A}+\nabla \varphi) \cdot \nabla \overline{\varphi^{\prime}} & =0 \text { in } \Omega_{c}
\end{aligned}
$$

where $V=X^{0}(\Omega) \times \widetilde{H^{1}}\left(\Omega_{c}\right)$ and :

$$
\begin{aligned}
X(\Omega)=H_{0}(\operatorname{rot}, \Omega) & =\left\{\mathbf{A} \in L^{2}(\Omega) ; \operatorname{rot} \mathbf{A} \in L^{2}(\Omega) \text { and } \mathbf{A} \times \mathbf{n}=\mathbf{0} \text { on } \Gamma\right\} \\
X^{0}(\Omega) & =\left\{\mathbf{A} \in X(\Omega) ;(\mathbf{A}, \nabla \xi)=0 \forall \xi \in H_{0}^{1}(\Omega)\right\} \\
\widetilde{H^{1}}\left(\Omega_{c}\right) & =\left\{\varphi \in H^{1}\left(\Omega_{c}\right) ; \int_{\Omega_{c}} \varphi=0\right\} .
\end{aligned}
$$

Let us note that the free-divergence Gauge condition on $\mathbf{A}$ is included in the definition of $X^{0}(\Omega)$, whereas the zero-mean value of $\varphi$ on $\Omega_{c}$ is included in the definition of $\widetilde{H^{1}}\left(\Omega_{c}\right)$. As it will be seen later on, these two conditions ensure the uniqueness of the solution 
$(\mathbf{A}, \varphi) \in V$. In the following, on a given domain $D$, the $L^{2}(D)$ norm will be denoted by $\|\cdot\|_{D}$, and the corresponding $L^{2}(D)$ scalar product by $(\cdot, \cdot)_{D}$. The usual norm and semi-norm of $H^{1}(D)$ will be denoted $\|\cdot\|_{1, D}$ and $|\cdot|_{1, D}$ respectively. In the case $D=\Omega$, we will drop the index $\Omega$. Now, $X(\Omega)$ is equipped with its usual norm :

$$
\|\mathbf{A}\|_{X(\Omega)}^{2}=\|\mathbf{A}\|^{2}+\|\operatorname{rot} \mathbf{A}\|^{2},
$$

and the natural norm $\|.\|_{V}$ associated with the Hilbert space $V$ is given by :

$$
\|(\mathbf{A}, \varphi)\|_{V}^{2}=\|\mathbf{A}\|_{X(\Omega)}^{2}+|\varphi|_{1, \Omega_{c}}^{2} .
$$

Multiplying (11) by $-j / \omega$ and adding this relation to (10), it can be directly obtained that an equivalent variational formulation to (10)-(11) is given by : Find $(\mathbf{A}, \varphi) \in V$ solution of

$$
a\left((\mathbf{A}, \varphi),\left(\mathbf{A}^{\prime}, \varphi^{\prime}\right)\right)=l\left(\left(\mathbf{A}^{\prime}, \varphi^{\prime}\right)\right), \forall\left(\mathbf{A}^{\prime}, \varphi^{\prime}\right) \in V,
$$

where $a$ and $l$ are respectively the following bilinear and linear forms defined by :

$$
\begin{aligned}
a\left((\mathbf{A}, \varphi),\left(\mathbf{A}^{\prime}, \varphi^{\prime}\right)\right) & =\int_{\Omega} \frac{1}{\mu} \operatorname{rot} \mathbf{A} \cdot \operatorname{rot} \overline{\mathbf{A}^{\prime}}-\int_{\Omega_{c}} \frac{j \sigma}{\omega}(j \omega \mathbf{A}+\nabla \varphi) \overline{\left(j \omega \mathbf{A}^{\prime}+\nabla \varphi^{\prime}\right)}, \\
l\left(\left(\mathbf{A}^{\prime}, \varphi^{\prime}\right)\right) & =\int_{\Omega} \mathbf{J}_{s} \cdot \overline{\mathbf{A}^{\prime}} .
\end{aligned}
$$

\subsection{Well-posedness of the problem}

Lemma 2.1 The bilinear form $\sqrt{2} e^{j \frac{\pi}{4}} a$ is coercive on $V$, namely there exists $C>0$ such that :

$$
\left|\sqrt{2} e^{j \frac{\pi}{4}} a((\mathbf{A}, \varphi),(\mathbf{A}, \varphi))\right| \geq C\|(\mathbf{A}, \varphi)\|_{V}^{2}, \quad \forall(\mathbf{A}, \varphi) \in V .
$$

Proof: First, let us notice that :

$$
\Re\left[\sqrt{2} e^{j \frac{\pi}{4}} a((\mathbf{A}, \varphi),(\mathbf{A}, \varphi))\right]=\int_{\Omega} \frac{1}{\mu}|\operatorname{rot} \mathbf{A}|^{2}+\int_{\Omega_{c}} \frac{\sigma}{\omega}|j \omega \mathbf{A}+\nabla \varphi|^{2} .
$$

Our aim is to prove that there exists $C>0$ such that for all $(\mathbf{A}, \varphi) \in V$,

$$
\int_{\Omega} \frac{1}{\mu}|\operatorname{rot} \mathbf{A}|^{2}+\int_{\Omega_{c}} \frac{\sigma}{\omega}|j \omega \mathbf{A}+\nabla \varphi|^{2} \geq C\|(\mathbf{A}, \varphi)\|_{V}^{2} .
$$

We proceed by using a contradiction argument. Let us suppose that there exists a sequence of $\left(\mathbf{A}_{n}, \varphi_{n}\right) \in V, n \in \mathbb{N}$, such that :

$$
\int_{\Omega} \frac{1}{\mu}\left|\operatorname{rot} \mathbf{A}_{n}\right|^{2}+\int_{\Omega_{c}} \frac{\sigma}{\omega}\left|j \omega \mathbf{A}_{n}+\nabla \varphi_{n}\right|^{2} \underset{n \rightarrow+\infty}{\longrightarrow} 0,
$$

as well as :

$$
\left\|\left(\mathbf{A}_{n}, \varphi_{n}\right)\right\|_{V}^{2}=1, \quad \forall n \in \mathbb{N} .
$$


Because of (13), we have :

$$
\left\|\operatorname{rot} \mathbf{A}_{n}\right\| \underset{n \rightarrow+\infty}{\longrightarrow} 0 .
$$

Since $\mathbf{A}_{n} \in X^{0}(\Omega)$, the Friedrichs-Poincaré inequality $\left\|\mathbf{A}_{n}\right\| \leq \tilde{C}\left\|\operatorname{rot} \mathbf{A}_{n}\right\|$ holds (see [16] p.88) and implies :

$$
\left\|\mathbf{A}_{n}\right\| \underset{n \rightarrow+\infty}{\longrightarrow} 0 \text {. }
$$

Moreover, the standard triangular inequality yields :

$$
\left\|\nabla \varphi_{n}\right\|_{\Omega_{c}} \leq\left\|\nabla \varphi_{n}-j \omega \mathbf{A}_{n}\right\|_{\Omega_{c}}+\omega\left\|\mathbf{A}_{n}\right\|_{\Omega_{c}} .
$$

Once again because of (13), we have :

$$
\int_{\Omega_{c}}\left|j \omega \mathbf{A}_{n}+\nabla \varphi_{n}\right|^{2} \underset{n \rightarrow+\infty}{\longrightarrow} 0
$$

From (16), (17) and (18), we conclude that:

$$
\left\|\nabla \varphi_{n}\right\|_{\Omega_{c}} \underset{n \rightarrow+\infty}{\longrightarrow} 0 .
$$

Clearly, (14) cannot hold because of (15), (16) and (19). This concludes the proof.

Theorem 2.2 The weak formulation (12) admits a unique solution $(\mathbf{A}, \varphi) \in V$.

Proof: The sesquilinear form $\sqrt{2} e^{j \frac{\pi}{4}} a$ is hermitian. It is obviously continuous on $V \times V$ and coercive on $V$ by Lemma 2.1. So Lax-Milgram's lemma ensures existence and uniqueness of a solution $(\mathbf{A}, \varphi) \in V$ to

$$
\sqrt{2} e^{j \frac{\pi}{4}} a\left((\mathbf{A}, \varphi),\left(\mathbf{A}^{\prime}, \varphi^{\prime}\right)\right)=\sqrt{2} e^{j \frac{\pi}{4}} l\left(\left(\mathbf{A}^{\prime}, \varphi^{\prime}\right)\right), \forall\left(\mathbf{A}^{\prime}, \varphi^{\prime}\right) \in V .
$$

Since $\sqrt{2} e^{j \frac{\pi}{4}}$ is different from zero, this problem is clearly equivalent to (12).

Now, we derive a very important result, coming from the free-divergence property of $\mathbf{J}_{s}$ on $\Omega$.

Lemma 2.3 Let $(\mathbf{A}, \varphi) \in V$ be the unique solution of (12). Then for all $\left(\mathbf{A}^{\prime}, \varphi^{\prime}\right) \in$ $X(\Omega) \times \widetilde{H}^{1}\left(\Omega_{c}\right)$, we have :

$$
a\left((\mathbf{A}, \varphi),\left(\mathbf{A}^{\prime}, \varphi^{\prime}\right)\right)=l\left(\left(\mathbf{A}^{\prime}, \varphi^{\prime}\right)\right) .
$$

Proof: Since $\mathbf{A}^{\prime} \in X(\Omega)$, we can decompose it using the following Helmholtz decomposition (see $[16$, p.66]) :

$$
\mathbf{A}^{\prime}=\Psi+\nabla \tau \text { with } \Psi \in X^{0}(\Omega) \text { and } \tau \in H_{0}^{1}(\Omega) .
$$

So we may write

$$
a\left((\mathbf{A}, \varphi),\left(\mathbf{A}^{\prime}, \varphi^{\prime}\right)\right)=a\left((\mathbf{A}, \varphi),\left(\Psi+\nabla \tau, \varphi^{\prime}\right)\right) .
$$


But we have

$$
\begin{aligned}
& a\left((\mathbf{A}, \varphi),\left(\Psi+\nabla \tau, \varphi^{\prime}\right)\right) \\
& =\int_{\Omega} \frac{1}{\mu} \operatorname{rot} \mathbf{A} \cdot \operatorname{rot} \bar{\Psi}-\int_{\Omega_{c}} \frac{j \sigma}{\omega}(j \omega \mathbf{A}+\nabla \varphi)\left(\overline{j \omega(\Psi+\nabla \tau)+\nabla \varphi^{\prime}}\right) \\
& =\int_{\Omega} \frac{1}{\mu} \operatorname{rot} \mathbf{A} \cdot \operatorname{rot} \bar{\Psi}-\int_{\Omega_{c}} \frac{j \sigma}{\omega}(j \omega \mathbf{A}+\nabla \varphi)\left(\overline{j \omega \Psi+\nabla\left(\varphi^{\prime}+j \omega \tau\right)}\right) \\
& =\int_{\Omega} \frac{1}{\mu} \operatorname{rot} \mathbf{A} \cdot \operatorname{rot} \bar{\Psi}-\int_{\Omega_{c}} \frac{j \sigma}{\omega}(j \omega \mathbf{A}+\nabla \varphi)\left(\overline{j \omega \Psi+\nabla\left(\varphi^{\prime}+j \omega \widetilde{\tau}\right)}\right),
\end{aligned}
$$

where we have defined :

$$
\widetilde{\tau}=\tau-\frac{1}{\left|\Omega_{c}\right|} \int_{\Omega_{c}} \tau, \quad \widetilde{\tau} \in \widetilde{H^{1}}\left(\Omega_{c}\right) .
$$

Hence by (12), we get

$$
\begin{aligned}
a\left((\mathbf{A}, \varphi),\left(\Psi+\nabla \tau, \varphi^{\prime}\right)\right) & =l\left(\left(\Psi, \varphi^{\prime}+j \omega \widetilde{\tau}\right)\right) \\
& =\left(\mathbf{J}_{s}, \Psi\right) \\
& =\left(\mathbf{J}_{s}, \mathbf{A}^{\prime}-\nabla \tau\right) .
\end{aligned}
$$

Using now the facts that $\operatorname{div} \mathbf{J}_{s}=0$ and $\tau \in H_{0}^{1}(\Omega)$, we conclude that :

$$
a\left((\mathbf{A}, \varphi),\left(\Psi+\nabla \tau, \varphi^{\prime}\right)\right)=\left(\mathbf{J}_{s}, \mathbf{A}^{\prime}\right)
$$

and (20) holds.

\subsection{Discrete formulation}

Now, the boundaries $B$ and $\Gamma$ are supposed to be polyhedral such that the domain $\Omega$ can be discretized by a conforming mesh $\mathscr{T}_{h}$ made of tetrahedra, each element $T$ of $\mathscr{T}_{h}$ belonging to either $\Omega_{c}$ or $\Omega_{e}$. The faces of $\mathscr{T}_{h}$ are denoted by $F$ and its edges by $E$. Let us note $h_{T}$ the diameter of $T$ and $\rho_{T}$ the diameter of its largest inscribed ball. We suppose that for any element $T$, the ratio $h_{T} / \rho_{T}$ is bounded by a constant $\alpha>0$ independant of $T$ and of the mesh size $h=\max _{T \in \mathscr{T}_{h}} h_{T}$. The set of faces (resp. edges and nodes) of the triangulation is denoted $\mathcal{F}$ (resp. $\mathcal{E}$ and $\mathcal{N})$, and we denote $h_{F}$ the diameter of the face $F$. The set of internal faces (resp. internal edges and internal nodes) to $\Omega$ is denoted $\mathcal{F}_{\text {int }}$ (resp. $\mathcal{E}_{\text {int }}$ and $\mathcal{N}_{\text {int }}$ ). The coefficients $\mu$ and $\sigma$ arising in (9) are moreover supposed to be constant on each tetrahedron of the mesh, and we will note $\mu_{T}=\mu_{\mid T}$ and $\sigma_{T}=\sigma_{\mid T}$ for all $T \in \mathscr{T}_{h}$. 
The approximation space $V_{h}$ is defined by $V_{h}=X_{h}^{0} \times \widetilde{\Theta}_{h}$, where :

$$
\begin{aligned}
& X_{h}=X(\Omega) \cap \mathcal{N D}_{1}\left(\Omega, \mathscr{T}_{h}\right)=\left\{\mathbf{A}_{h} \in X(\Omega) ; \mathbf{A}_{h \mid T} \in \mathcal{N D}_{1}(T), \forall T \in \mathscr{T}_{h}\right\}, \\
& \mathcal{N D}_{1}(T)=\left\{A_{h}: \begin{array}{ccc}
T & \longrightarrow & \mathbb{C}^{3} \\
\mathbf{x} & \longrightarrow \mathbf{a}+\mathbf{b} \times \mathbf{x}
\end{array}, \mathbf{a}, \mathbf{b} \in \mathbb{C}^{3}\right\}, \\
& \Theta_{h}^{0}=\left\{\xi_{h} \in H_{0}^{1}(\Omega) ; \xi_{h \mid T} \in \mathbb{P}_{1}(T) \forall T \in \mathscr{T}_{h}\right\}, \\
& X_{h}^{0}=\left\{\mathbf{A}_{h} \in X_{h} ;\left(\mathbf{A}_{h}, \nabla \xi_{h}\right)=0 \forall \xi_{h} \in \Theta_{h}^{0}\right\} \text {, } \\
& \widetilde{\Theta}_{h}=\left\{\varphi_{h} \in \widetilde{H^{1}}\left(\Omega_{c}\right) ; \varphi_{h \mid T} \in \mathbb{P}_{1}(T) \forall T \in \mathscr{T}_{h}\right\} .
\end{aligned}
$$

Now, the discretized weak formulation is given by : Find $\left(\mathbf{A}_{h}, \varphi_{h}\right) \in V_{h}$ such that for all $\left(\mathbf{A}_{h}^{\prime}, \varphi_{h}^{\prime}\right) \in V_{h}$ we have :

$$
a\left(\left(\mathbf{A}_{h}, \varphi_{h}\right),\left(\mathbf{A}_{h}^{\prime}, \varphi_{h}^{\prime}\right)\right)=l\left(\left(\mathbf{A}_{h}^{\prime}, \varphi_{h}^{\prime}\right)\right) .
$$

Theorem 2.4 The weak formulation (21) admits one unique solution $\left(\mathbf{A}_{h}, \varphi_{h}\right) \in V_{h}$.

Proof: The proof is in any point similar to the one of Theorem 2.2 in the continuous case. The main point relies in proving the coercivity of $a$ on $V_{h}$, which can be done by using the discrete Poincaré-Friedrichs inequality $\left\|\mathbf{A}_{h}\right\| \leq C \|$ rot $\mathbf{A}_{h} \|$ for all $\mathbf{A}_{h} \in X_{h}^{0}$, with $C>0$ independent of $h$, see [16] p. 185, Lemma 7.20 for all details.

Remark 2.5 Let us notice that, because of the discrete Gauge condition arising in the definition of $X_{h}^{0}, V_{h}$ is not included in $V$, so that the approximation is not a conforming one. Nevertheless, the free-divergence property of $\mathbf{J}_{s}$ on $\Omega$ gives rise to a discrete property similar to the continuous one given in Lemma 2.3.

Lemma 2.6 For all $\left(\mathbf{A}_{h}^{\prime}, \varphi_{h}^{\prime}\right) \in X_{h} \times \widetilde{\Theta}_{h}$, we have :

$$
a\left(\left(\mathbf{A}_{h}, \varphi_{h}\right),\left(\mathbf{A}_{h}^{\prime}, \varphi_{h}^{\prime}\right)\right)=l\left(\left(\mathbf{A}_{h}^{\prime}, \varphi_{h}^{\prime}\right)\right) .
$$

Proof:: Since $\mathbf{A}_{h}^{\prime} \in X_{h}$, this time we can use the discrete Helmholtz decomposition [23, p. 272]:

$$
\mathbf{A}_{h}^{\prime}=\Psi_{h}+\nabla \tau_{h} \text { with } \Psi_{h} \in X_{h}^{0} \text { and } \tau_{h} \in \Theta_{h}^{0} .
$$

The proof now follows the one of the continuous case, see Lemma 2.3.

A direct consequence of Lemmas 2.3 and 2.6 is the following orthogonality property, despite the fact that the approximation is not a conforming one :

Lemma 2.7 For all $\left(\mathbf{A}_{h}^{\prime}, \varphi_{h}^{\prime}\right) \in X_{h} \times \widetilde{\Theta}_{h}$, we have :

$$
a\left(\left(\mathbf{A}-\mathbf{A}_{h}, \varphi-\varphi_{h}\right),\left(\mathbf{A}_{h}^{\prime}, \varphi_{h}^{\prime}\right)\right)=0 .
$$




\section{Analytical tools}

Here, we develop or recall some analytical tools which will be very useful in section 4 devoted to the derivation of the a posteriori error estimators. In the following, the notations $a \lesssim b$ and $a \sim b$ mean the existence of positive constants $C_{1}$ and $C_{2}$ which are independent of the quantities $a$ and $b$ under consideration as well as of the mesh size $h$, the coefficients $\mu, \sigma$ and of the frequency $\omega$, such that $a \leq C_{2} b$ and $C_{1} b \leq a \leq C_{2} b$, respectively.

\subsection{Helmholtz decomposition}

Let us first respectively define the errors on $\mathbf{A}$ and $\varphi$ by :

$$
\mathbf{e}_{A}=\mathbf{A}-\mathbf{A}_{h} \in X(\Omega)
$$

and

$$
e_{\varphi}=\varphi-\varphi_{h} \in \widetilde{H^{1}}\left(\Omega_{c}\right)
$$

We also define $e_{\varphi}^{(e)} \in H^{1}\left(\Omega_{e}\right)$ by :

$$
\left\{\begin{array}{l}
\Delta e_{\varphi}^{(e)}=0 \quad \text { in } \Omega_{e} \\
e_{\varphi}^{(e)} \mid B=e_{\varphi \mid B} \\
e_{\varphi}^{(e)}=0
\end{array}\right.
$$

and $\widetilde{e_{\varphi}} \in H_{0}^{1}(\Omega)$ by :

$$
\tilde{e_{\varphi}}=\left\{\begin{array}{cc}
e_{\varphi} & \text { in } \Omega_{c} \\
e_{\varphi}^{(e)} & \text { in } \Omega_{e}
\end{array}\right.
$$

Theorem 3.1 The error $j \omega \mathbf{e}_{A}+\nabla \widetilde{e_{\varphi}}$ admits the following Helmholtz decomposition :

$$
j \omega \mathbf{e}_{A}+\nabla \tilde{e_{\varphi}}=\nabla \hat{\varphi}+\mathbf{e}_{\perp}
$$

where $\hat{\varphi} \in H_{0}^{1}(\Omega)$ and $e_{\perp} \in X^{0}(\Omega)$, with:

$$
\begin{gathered}
|\hat{\varphi}|_{1, \Omega_{c}} \lesssim\left\|j \omega \mathbf{e}_{A}+\nabla e_{\varphi}\right\|_{\Omega_{c}} \\
\left\|\mathbf{e}_{\perp}\right\|_{X(\Omega)} \lesssim\left\|j \omega \mathbf{e}_{A}+\nabla e_{\varphi}\right\|_{\Omega_{c}}+\omega\left\|\operatorname{rot} \mathbf{e}_{A}\right\| .
\end{gathered}
$$

Moreover, we have :

$$
\mathbf{e}_{\perp}=\nabla \Phi+\mathbf{w}
$$

where $\mathbf{w}_{c}=\mathbf{w}_{\mid \Omega_{c}} \in H^{1}\left(\Omega_{c}\right)^{3}, \mathbf{w}_{e}=\mathbf{w}_{\mid \Omega_{e}} \in H^{1}\left(\Omega_{e}\right)^{3}$, and $\Phi \in H_{0}^{1}(\Omega)$ are such that :

$$
\begin{aligned}
|\Phi|_{1} & \lesssim\left\|\mathbf{e}_{\perp}\right\|_{X(\Omega)}, \\
\left(\left\|\mathbf{w}_{c}\right\|_{1, \Omega_{c}}^{2}+\left\|\mathbf{w}_{e}\right\|_{1, \Omega_{e}}^{2}\right)^{1 / 2} & \lesssim\left\|\mathbf{e}_{\perp}\right\|_{X(\Omega)} .
\end{aligned}
$$


Proof: First of all, let us define $\hat{\varphi}^{(c)} \in \widetilde{H^{1}}\left(\Omega_{c}\right)$ such that :

$$
\left\{\begin{array}{rll}
\operatorname{div} \nabla \hat{\varphi}^{(c)} & =\operatorname{div}\left(j \omega \mathbf{e}_{A}+\nabla e_{\varphi}\right) & \text { in } \Omega_{c} \\
\nabla \hat{\varphi}^{(c)} \cdot \mathbf{n} & =\left(j \omega \mathbf{e}_{A}+\nabla e_{\varphi}\right) \cdot \mathbf{n} & \text { on } B
\end{array}\right.
$$

where $\mathbf{n}$ is the outward unit normal to $\Omega_{c}$ on $B$. Clearly,

$$
\left|\hat{\varphi}^{(c)}\right|_{1, \Omega_{c}} \leq\left\|j \omega \mathbf{e}_{A}+\nabla e_{\varphi}\right\|_{\Omega_{c}}
$$

We define $\mathbf{e}_{\perp}^{(c)}=j \omega \mathbf{e}_{A}+\nabla e_{\varphi}-\nabla \hat{\varphi}^{(c)}$ in $\Omega_{c}$. From $(30), \operatorname{div} \mathbf{e}_{\perp}^{(c)}=0$ on $\Omega_{c}$ and $\mathbf{e}_{\perp}^{(c)} \cdot \mathbf{n}=0$ on $B$, so that:

$$
\int_{\Omega_{c}} \nabla \hat{\varphi}^{(c)} \cdot \overline{\mathbf{e}_{\perp}^{(c)}}=-\int_{\Omega_{c}} \hat{\varphi}^{(c)} \cdot \operatorname{div} \overline{\mathbf{e}_{\perp}^{(c)}}+\int_{B} \hat{\varphi}^{(c)} \cdot \overline{\mathbf{e}_{\perp}^{(c)} \cdot \mathbf{n}}=0,
$$

and therefore

$$
\left\|j \omega \mathbf{e}_{A}+\nabla e_{\varphi}\right\|_{\Omega_{c}}^{2}=\left|\hat{\varphi}^{(c)}\right|_{1, \Omega_{c}}^{2}+\left\|\mathbf{e}_{\perp}^{(c)}\right\|_{\Omega_{c}}^{2}
$$

Consequently,

$$
\left\|\mathbf{e}_{\perp}^{(c)}\right\|_{\Omega_{c}} \leq\left\|j \omega \mathbf{e}_{A}+\nabla e_{\varphi}\right\|_{\Omega_{c}}
$$

Then, $\hat{\hat{\varphi}} \in H^{1}\left(\Omega_{e}\right)$ is defined as the unique weak solution of

$$
\left\{\begin{aligned}
\operatorname{div} \nabla \hat{\hat{\varphi}} & =\operatorname{div}\left(j \omega \mathbf{e}_{A}+\nabla \tilde{e_{\varphi}}\right) & & \text { in } \Omega_{e}, \\
\left.\hat{\hat{\varphi}}\right|_{\Omega_{e}} & =\left.\hat{\varphi}^{(c)}\right|_{\Omega_{c}} & & \text { on } B \\
\hat{\hat{\varphi}} & =0 & & \text { on } \Gamma .
\end{aligned}\right.
$$

Now fix $\psi \in H^{1}\left(\Omega_{e}\right)$ as the unique weak solution of

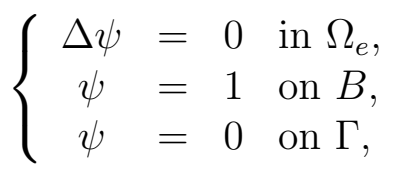

and define $\hat{\varphi}^{(e)}=\hat{\hat{\varphi}}+k \psi$ on $\Omega_{e}$ where $k$ is chosen by :

$$
k=\frac{\int_{B}\left(j \omega \mathbf{e}_{A}+\nabla \tilde{e_{\varphi}}\right) \cdot \mathbf{n}-\int_{B} \nabla \hat{\hat{\varphi}} \cdot \mathbf{n}}{\int_{B} \nabla \psi \cdot \mathbf{n}} .
$$

Note that $\int_{B} \nabla \psi \cdot \mathbf{n}>0$ because by Green's formula and (34), we see that

$$
\int_{B} \nabla \psi \cdot \mathbf{n}=\int_{B} \nabla \psi \cdot \mathbf{n} \psi=\int_{\Omega_{e}}|\nabla \psi|^{2} .
$$


From (33) and (34) we have :

$$
\left\{\begin{aligned}
\operatorname{div} \nabla \hat{\varphi}^{(e)} & =\operatorname{div}\left(j \omega \mathbf{e}_{A}+\nabla \widetilde{e_{\varphi}}\right) & & \text { in } \Omega_{e}, \\
\left.\hat{\varphi}^{(e)}\right|_{\Omega_{e}} & =\hat{\varphi}^{(c)}+k & & \text { on } B, \\
\hat{\varphi}^{(e)} & =0 & & \text { on } \Gamma .
\end{aligned}\right.
$$

Let us define $\mathbf{e}_{\perp}^{(e)}=j \omega \mathbf{e}_{A}+\nabla \widetilde{e_{\varphi}}-\nabla \hat{\varphi}^{(e)}$ in $\Omega_{e}$, then we have :

$$
\int_{B} \mathbf{e}_{\perp}^{(e)} \cdot \mathbf{n}=\int_{B}\left(j \omega \mathbf{e}_{A}+\nabla \widetilde{e_{\varphi}}\right) \cdot \mathbf{n}-\int_{B} \nabla \hat{\hat{\varphi}} \cdot \mathbf{n}-k \int_{B} \nabla \psi \cdot \mathbf{n}
$$

And, from the choice (35), we can conclude that :

$$
\int_{B} \mathbf{e}_{\perp}^{(e)} \cdot \mathbf{n}=0
$$

If we define now $\mathbf{e}_{\perp} \in X(\Omega)$ by :

$$
\mathbf{e}_{\perp}= \begin{cases}\mathbf{e}_{\perp}^{(c)} & \text { in } \Omega_{c}, \\ \mathbf{e}_{\perp}^{(e)} & \text { in } \Omega_{e},\end{cases}
$$

we have clearly proved that $\mathbf{e}_{\perp} \in Y(\Omega)$, where $Y(\Omega)$ is defined by :

$$
Y(\Omega)=\left\{\mathbf{u} \in X(\Omega) ; \operatorname{div} \mathbf{u}_{\mid \Omega_{c}} \in L^{2}\left(\Omega_{c}\right), \operatorname{div} \mathbf{u}_{\mid \Omega_{e}} \in L^{2}\left(\Omega_{e}\right), \quad \int_{B} \mathbf{u}_{\mid \Omega_{e}} \cdot \mathbf{n}=0\right\} .
$$

From Lemma 2.2 of [9], we obtain :

$$
\left\|\mathbf{e}_{\perp}^{(e)}\right\|_{\Omega_{e}} \lesssim\left\|\operatorname{rot} \mathbf{e}_{\perp}\right\|+\left\|\mathbf{e}_{\perp}^{(c)}\right\|_{\Omega_{c}} .
$$

From (32) and using the fact that $\left\|\operatorname{rot} \mathbf{e}_{\perp}\right\|=\omega\left\|\operatorname{rot} \mathbf{e}_{A}\right\|$, we get :

$$
\left\|\mathbf{e}_{\perp}^{(e)}\right\|_{\Omega_{e}} \lesssim \omega\left\|\operatorname{rot} \mathbf{e}_{A}\right\|+\left\|j \omega \mathbf{e}_{A}+\nabla e_{\varphi}\right\|_{\Omega_{c}} .
$$

Finally, defining $\hat{\varphi} \in H_{0}^{1}(\Omega)$ by :

$$
\hat{\varphi}=\left\{\begin{array}{cc}
\hat{\varphi}^{(c)}+k & \text { in } \Omega_{c}, \\
\hat{\varphi}^{(e)} & \text { in } \Omega_{e},
\end{array}\right.
$$

we derive (25) from (31), and (26) from (32) and (36).

Now it remains to prove (27), (28) and (29). For that purpose, let us recall that by construction, $\mathbf{e}_{\perp} \in \tilde{Y}(\Omega)$ where :

$$
\tilde{Y}(\Omega)=\left\{\mathbf{u} \in X(\Omega) ; \operatorname{div} \mathbf{u}_{\mid \Omega_{c}} \in L^{2}\left(\Omega_{c}\right), \operatorname{div} \mathbf{u}_{\mid \Omega_{e}} \in L^{2}\left(\Omega_{e}\right), \text { and } \mathbf{u}_{\mid \Omega_{c}} \cdot \mathbf{n}=0 \text { on } B\right\} .
$$

Consequently, Theorem 3.1 of [9] yields :

$$
\mathbf{e}_{\perp}=\nabla \Phi+\mathbf{w}
$$

with $\mathbf{w} \in \tilde{Y}(\Omega)$ is such that $\mathbf{w}_{c}=\mathbf{w}_{\mid \Omega_{c}} \in H^{1}\left(\Omega_{c}\right)^{3}, \mathbf{w}_{e}=\mathbf{w}_{\mid \Omega_{e}} \in H^{1}\left(\Omega_{e}\right)^{3}, \Phi \in H_{0}^{1}(\Omega)$ with :

$$
|\Phi|_{1}+\left(\left\|\mathbf{w}_{c}\right\|_{1, \Omega_{c}}^{2}+\left\|\mathbf{w}_{e}\right\|_{1, \Omega_{e}}^{2}\right)^{1 / 2} \lesssim\left\|\mathbf{e}_{\perp}\right\|_{X(\Omega)} .
$$




\subsection{Standard Clément interpolation operator}

For our further analysis, we need an interpolation operator that maps a function from $H_{0}^{1}(\Omega)$ to $\Theta_{h}^{0}$, as well as an interpolation operator that maps a function from $H^{1}\left(\Omega_{c}\right)$ to $\Theta_{h}\left(\Omega_{c}\right)=\left\{\xi_{h} \in H^{1}\left(\Omega_{c}\right) ; \xi_{h \mid T} \in \mathbb{P}_{1}(T) \forall T \in \mathscr{T}_{h} \cap \Omega_{c}\right\}$. Hence Lagrange interpolation is unsuitable, but Clément like interpolant is more appropriate. Recall that the nodal basis functions $\varphi_{\mathbf{x}} \in \Theta_{h}^{0}$ associated with a node $\mathbf{x}$ is uniquely determined by the condition :

$$
\varphi_{\mathbf{x}}(\mathbf{y})=\delta_{\mathbf{x}, \mathbf{y}}, \quad \forall \mathbf{y} \in \mathcal{N} .
$$

Moreover, for any $x \in \mathcal{N}$, we define $\omega_{\mathrm{x}}$ as the set of tetrahedra containing the node $\mathbf{x}$.

Definition 3.2 We define the Clément interpolation operator $I_{C l}^{0}: H_{0}^{1}(\Omega) \rightarrow \Theta_{h}^{0}$ by :

$$
I_{C l}^{0} v=\sum_{\mathbf{x} \in \mathcal{N}_{i n t}} \frac{1}{\left|\omega_{\mathbf{x}}\right|}\left(\int_{\omega_{\mathbf{x}}} v\right) \varphi_{\mathbf{x}},
$$

where $\omega_{\mathbf{x}}$ is the set of tetrahedra containing the node $\mathbf{x}$.

Definition 3.3 We define the Clément interpolation operator $I_{C l}: H^{1}\left(\Omega_{c}\right) \rightarrow \Theta_{h}\left(\Omega_{c}\right)$ by :

$$
I_{C l} v=\sum_{\mathbf{x} \in \mathcal{N} \cap \overline{\Omega_{c}}} \frac{1}{\left|\omega_{\mathbf{x}} \cap \Omega_{c}\right|}\left(\int_{\omega_{\mathbf{x}} \cap \Omega_{c}} v\right) \varphi_{\mathbf{x}},
$$

Then, we can state the following usual interpolation estimates :

Lemma 3.4 For any $v^{0} \in H_{0}^{1}(\Omega)$ and $v \in H^{1}\left(\Omega_{c}\right)$ it holds :

$$
\begin{aligned}
& \sum_{T \in \mathscr{T}_{h}} h_{T}^{-2}\left\|v^{0}-I_{C l}^{0} v^{0}\right\|_{T}^{2} \lesssim\left\|\nabla v^{0}\right\|^{2}, \\
& \sum_{F \in \mathcal{F}_{i n t}} h_{F}^{-1}\left\|v^{0}-I_{C l}^{0} v^{0}\right\|_{F}^{2} \lesssim\left\|\nabla v^{0}\right\|^{2}, \\
& \sum_{T \in \mathscr{T}_{h}, T \subset \Omega_{c}} h_{T}^{-2}\left\|v-I_{C l} v\right\|_{T}^{2} \lesssim\|\nabla v\|_{\Omega_{c}}^{2}, \\
& \sum_{F \in \mathcal{F}, F \subset \overline{\Omega_{c}}} h_{F}^{-1}\left\|v-I_{C l} v\right\|_{F}^{2} \lesssim\|\nabla v\|_{\Omega_{c}}^{2} .
\end{aligned}
$$

Proof: See [7]. 


\subsection{Vectorial Clément-type interpolation operator}

Since our problem also involves functions in $X(\Omega)$, we further need a Clément-type interpolant mapping a (vector) function in $X(\Omega)$ to $X_{h}$. This operator was introduced in [19] in an anisotropic context (for an isotropic version, see [3]), we recall it here for completeness. It is defined with the help of the basis functions $\mathbf{w}_{E} \in X_{h}, E \in \mathcal{E}$, defined by the condition :

$$
\int_{E^{\prime}} \mathbf{w}_{E} \cdot \mathbf{t}_{E^{\prime}}=\delta_{\mathbf{E}, \mathbf{E}}, \quad \forall E^{\prime} \in \mathcal{E}
$$

where $\mathbf{t}_{E}$ means the unit vector directed along $E$. Let us define $P H^{1}(\Omega)$ as the set of functions which are piecewise $H^{1}$ on the domain $\Omega$ (with respect to the splitting of so called "broken gradient" associated with this decomposition.

Definition 3.5 For any edge $E \in \mathcal{E}$ fix one of its adjacent faces that we call $F_{E} \in \mathcal{F}$. Then define the Clément type interpolation operator $\mathcal{P}_{C l}:\left[P H^{1}(\Omega)\right] \cap X(\Omega) \rightarrow X_{h}$ by :

$$
\mathcal{P}_{C l} \mathbf{v}=\sum_{E \in \mathcal{E}}\left(\int_{F_{E}}\left(\mathbf{v} \times \mathbf{n}_{F_{E}}\right) \cdot \mathbf{f}_{E}^{F_{E}}\right) \mathbf{w}_{E},
$$

where the (vector) functions $\mathbf{f}_{E^{\prime}}^{F_{E}}$ are determined by the condition :

$$
\int_{F_{E}}\left(\mathbf{w}_{E^{\prime}} \times \mathbf{n}_{F_{E}}\right) \cdot \mathbf{f}_{E^{\prime \prime}}^{F_{E}}=\delta_{E^{\prime}, E^{\prime \prime}}, \quad \forall E^{\prime}, E^{\prime \prime} \in \mathcal{E} \cup \partial \mathcal{F}_{\mathcal{E}} .
$$

Then, we can state the following usual interpolation estimates :

Lemma 3.6 For all $\mathbf{v} \in\left[P H^{1}(\Omega)\right] \cap X(\Omega)$, we have :

$$
\begin{aligned}
\sum_{T \in \mathscr{T}_{h}} h_{T}^{-2}\left\|\mathbf{v}-\mathcal{P}_{C l} \mathbf{v}\right\|_{T}^{2} & \lesssim\left\|\nabla_{P} \mathbf{v}\right\|^{2} \\
\sum_{F \in \mathcal{F}} h_{F}^{-1}\left\|\mathbf{v}-\mathcal{P}_{C l} \mathbf{v}\right\|_{F}^{2} & \lesssim\left\|\nabla_{P} \mathbf{v}\right\|^{2}
\end{aligned}
$$

Proof: See [3].

\subsection{Bubble functions and inverse inequalities}

For the analysis we finally require bubble functions and extension operators that satisfy certain properties. On the reference element $\bar{T}$ whose vertices are $\{(0,0,0) ;(1,0,0) ;(0,1,0) ;(0,0,1)\}$, we define the element bubble function $b_{\bar{T}} \in C(\bar{T})$ as $b_{\bar{T}}(\bar{x}, \bar{y}, \bar{z}):=4^{4} \bar{x} \bar{y} \bar{z}(1-\bar{x}-\bar{y}-\bar{z})$. We also need a face bubble function $b_{\bar{F}, \bar{T}} \in C(\bar{T})$ for a face $\bar{F} \subset \partial \bar{T}$. Without loss of generality assume that $\bar{F}$ is in the $\bar{x} \bar{y}$ plane and then take $b_{\bar{F}, \bar{T}}(\bar{x}, \bar{y}, \bar{z}):=3^{3} \bar{x} \bar{y}(1-\bar{x}-\bar{y}-\bar{z})$. Furthermore the next extension operator $\mathrm{F}_{\text {ext }}: C(\bar{F}) \rightarrow C(\bar{T})$ will be necessary $\mathrm{F}_{\text {ext }}\left(v_{\bar{F}}\right)(\bar{x}, \bar{y}, \bar{z}):=$ $v_{\bar{F}}(\bar{x}, \bar{y})$. 
The element bubble function $b_{T}$ for the actual element $T$ is obtained simply by the corresponding affine linear transformation. Similarly the face bubble function $b_{F, T}$ is defined. Later on a face bubble function $b_{F}$ is needed on the domain $\omega_{F}=T_{1} \cup T_{2}$ with $F=\bar{T}_{1} \cap \bar{T}_{2}$. This is achieved by an elementwise definition, i.e.

$$
\left.b_{F}\right|_{T_{i}}:=b_{F, T_{i}}, \quad i=1,2 .
$$

Analogously the extension operator is defined for functions $v_{F} \in C(F)$. By the same elementwise definition we obtain that $\mathrm{F}_{\text {ext }}\left(v_{F}\right) \in C\left(\omega_{F}\right)$. With these definitions one easily checks

$$
b_{T}=0 \text { on } \partial T, \quad b_{F}=0 \text { on } \partial \omega_{F}, \quad\left\|b_{T}\right\|_{\infty, T}=\left\|b_{F}\right\|_{\infty, \omega_{F}}=1 .
$$

Next, one requires so-called inverse inequalities that only hold in some finite dimensional spaces. The choice $\mathbb{P}^{k}$ covers all relevant cases of our analysis.

Lemma 3.7 (Inverse inequalities) Let $v_{T} \in \mathbb{P}^{k_{0}}(T)$ and $v_{F} \in \mathbb{P}^{k_{1}}(F)$. Then the following equivalences/inequalities hold. The inequality constants depend on the polynomial degree $k_{0}$ or $k_{1}$ but not on $T, E$ or $v_{T}, v_{F}$.

$$
\begin{aligned}
\left\|v_{T}\right\|_{T} & \lesssim\left\|v_{T} b_{T}^{1 / 2}\right\|_{T} \\
\left\|\nabla\left(v_{T} b_{T}\right)\right\|_{T} & \lesssim h_{T}^{-1}\left\|v_{T}\right\|_{T} \\
\left\|v_{F}\right\|_{F} & \lesssim\left\|v_{F} b_{F}^{1 / 2}\right\|_{F} \\
\left\|\mathrm{~F}_{\mathrm{ext}}\left(v_{F}\right) b_{F}\right\|_{T} & \lesssim h_{F}^{1 / 2}\left\|v_{F}\right\|_{F} \\
\left\|\nabla\left(\mathrm{F}_{\mathrm{ext}}\left(v_{F}\right) b_{F}\right)\right\|_{T} & \lesssim h_{F}^{-1 / 2}\left\|v_{F}\right\|_{F}
\end{aligned}
$$

Proof: See [24].

\section{A posteriori error estimation}

\subsection{Definition of the residual}

For all $\left(\mathbf{A}^{\prime}, \varphi^{\prime}\right) \in X(\Omega) \times \widetilde{H^{1}}\left(\Omega_{c}\right)$, we define the residual by :

$$
r\left(\mathbf{A}^{\prime}, \varphi^{\prime}\right)=\left(\mathbf{J}_{s}, \mathbf{A}^{\prime}\right)-a\left(\left(\mathbf{A}_{h}, \varphi_{h}\right),\left(\mathbf{A}^{\prime}, \varphi^{\prime}\right)\right) .
$$

Lemma 4.1 Let us recall that $\mathbf{e}_{A}, \mathbf{e}_{\varphi}$ and $\tilde{\mathbf{e}_{\varphi}}$ are respectively defined by (22), (23) and (24). Then,

$$
r\left(\mathbf{e}_{A}, e_{\varphi}\right)=r\left(-\frac{j}{\omega}\left(j \omega \mathbf{e}_{A}+\nabla \widetilde{e_{\varphi}}\right), 0\right) .
$$

Proof: The proof is based on the facts that $\widetilde{e_{\varphi}} \in H_{0}^{1}(\Omega), \widetilde{e_{\varphi} \mid \Omega_{c}}=e_{\varphi}$, and $\operatorname{div} \mathbf{J}_{s}=0$ in $\Omega$. 
Lemma 4.2 We have :

$$
\sqrt{2} \Re\left(e^{j \pi / 4} r\left(\mathbf{e}_{A}, e_{\varphi}\right)\right)=\int_{\Omega} \frac{1}{\mu}\left|\operatorname{rot} \mathbf{e}_{A}\right|^{2}+\int_{\Omega_{c}} \frac{\sigma}{\omega}\left|j \omega \mathbf{e}_{A}+\nabla e_{\varphi}\right|^{2}
$$

Proof:

$$
r\left(\mathbf{e}_{A}, e_{\varphi}\right)=\left(\mathbf{J}_{s}, \mathbf{A}-\mathbf{A}_{h}\right)-a\left(\left(\mathbf{A}_{h}, \varphi_{h}\right),\left(\mathbf{A}-\mathbf{A}_{h}, \varphi-\varphi_{h}\right)\right) .
$$

Since $\mathbf{A}-\mathbf{A}_{h} \in X(\Omega)$ and $\varphi-\varphi_{h} \in \widetilde{H^{1}}\left(\Omega_{c}\right)$, Lemma 2.3 leads to :

$$
\left(\mathbf{J}_{s}, \mathbf{A}-\mathbf{A}_{h}\right)=a\left((\mathbf{A}, \varphi),\left(\mathbf{A}-\mathbf{A}_{h}, \varphi-\varphi_{h}\right)\right) .
$$

Finally, (49) and (50) yield :

$$
r\left(\mathbf{e}_{A}, e_{\varphi}\right)=a\left(\left(\mathbf{e}_{A}, e_{\varphi}\right),\left(\mathbf{e}_{A}, e_{\varphi}\right)\right),
$$

and (48) holds.

Now, we are interested in deriving an a posteriori error estimator in order to control the error defined as the right-hand side of (48). Consequently, from Lemmas 4.1 and 4.2, we are reduced to bound from above the quantity $\left|r\left(-\frac{j}{\omega}\left(j \omega \mathbf{e}_{A}+\nabla \tilde{e_{\varphi}}\right), 0\right)\right|$.

\subsection{Definition of the estimators}

Definition 4.3 The local error estimator on the thetraedron $T$ is defined by:

$$
\eta_{T}^{2}=\eta_{T ; 1}^{2}+\eta_{T ; 2}^{2}+\eta_{T ; 3}^{2}+\sum_{F \subset \partial T}\left(\eta_{F ; 1}^{2}+\eta_{F ; 2}^{2}\right)
$$

with :

$$
\begin{aligned}
& \eta_{T ; 1}=h_{T}\left\|\pi_{h} \mathbf{J}_{s}-\operatorname{rot}\left(\frac{1}{\mu} \operatorname{rot} \mathbf{A}_{h}\right)-\sigma\left(j \omega \mathbf{A}_{h}+\nabla \varphi_{h}\right)\right\|_{T}, \\
& \eta_{T ; 2}=h_{T}\left\|\mathbf{J}_{s}-\pi_{h} \mathbf{J}_{s}\right\|_{T}, \\
& \eta_{T ; 3}=h_{T}\left\|\operatorname{div}\left(\sigma\left(j \omega \mathbf{A}_{h}+\nabla \varphi_{h}\right)\right)\right\|_{T}, \\
& \eta_{F ; 1}=h_{F}^{1 / 2}\left\|\left[\mathbf{n} \times \frac{1}{\mu} \operatorname{rot} \mathbf{A}_{h}\right]_{F}\right\|_{F}, \\
& \eta_{F ; 2}=h_{F}^{1 / 2}\left\|\left[\sigma\left(j \omega \mathbf{A}_{h}+\nabla \varphi_{h}\right) \cdot \mathbf{n}\right]_{F}\right\|_{F} .
\end{aligned}
$$

Moreover, the global error estimator is defined by :

$$
\eta^{2}=\sum_{T \in \mathcal{T}} \eta_{T}^{2} .
$$


Here, $\pi_{h}$ stands for the projection operator from $H(\operatorname{div}, \Omega)=\left\{\mathbf{u} \in L^{2}(\Omega) ; \operatorname{div} \mathbf{u} \in L^{2}(\Omega)\right\}$ to an ad hoc discrete approximation space and $[\mathbf{u}]_{F}$ denotes the jump of the quantity $\mathbf{u}$ through the face $F$ of the mesh.

Remark 4.4 Practically, $\eta_{T ; 2}$ corresponds to a superconvergent negligible contribution in the estimator. Consequently, we could choose to replace it by a generic quantity h.o.t corresponding to a higher order term.

Remark 4.5 Because of the chosen low-order discretization spaces (see definitions of $X_{h}^{0}$ and $\widetilde{\Theta_{h}}$ above), we clearly have $\eta_{T ; 3}=0$. Nevertheless, this term would not vanish if higher discretization spaces would be used.

\subsection{Reliability}

Theorem 4.6 We have :

$$
\left(\int_{\Omega} \frac{1}{\mu}\left|\operatorname{rot} \mathbf{e}_{A}\right|^{2}+\int_{\Omega_{c}} \frac{\sigma}{\omega}\left|j \omega \mathbf{e}_{A}+\nabla e_{\varphi}\right|^{2}\right)^{1 / 2} \lesssim C_{u p} \eta,
$$

with

$$
C_{u p}=\max \left\{\max _{T \in \Omega} \mu_{T}^{1 / 2}, \frac{1}{\omega^{1 / 2}} \max _{T \in \Omega_{c}}\left\{\frac{1}{\sigma_{T}^{1 / 2}}\right\}\right\} .
$$

Proof: To begin with, we use the Helmholtz decomposition with $\hat{\varphi}$, w and $\Phi$ given by Theorem 3.1 :

$$
j \omega \mathbf{e}_{A}+\nabla \widetilde{e_{\varphi}}=\nabla \hat{\varphi}+\mathbf{w}+\nabla \Phi .
$$

We have from Lemma 4.1:

$$
\begin{aligned}
\omega\left|r\left(\mathbf{e}_{A}, e_{\varphi}\right)\right|= & \mid \int_{\Omega} \mathbf{J}_{s} \cdot \overline{-j(\nabla \hat{\varphi}+\mathbf{w}+\nabla \Phi)}-\int_{\Omega} \frac{1}{\mu} \operatorname{rot} \mathbf{A}_{h} \cdot \operatorname{rot} \overline{-j(\nabla \hat{\varphi}+\mathbf{w}+\nabla \Phi)} \\
& +\int_{\Omega_{c}} j \sigma\left(j \omega \mathbf{A}_{h}+\nabla \varphi_{h}\right) \overline{(\nabla \hat{\varphi}+\mathbf{w}+\nabla \Phi)} \mid \\
= & \mid \int_{\Omega} \mathbf{J}_{s} \cdot \overline{-j \mathbf{w}}-\int_{\Omega} \frac{1}{\mu} \operatorname{rot} \mathbf{A}_{h} \cdot \operatorname{rot} \overline{-j \mathbf{w}}+\int_{\Omega_{c}} j \sigma\left(j \omega \mathbf{A}_{h}+\nabla \varphi_{h}\right) \overline{\mathbf{w}} \\
& +\int_{\Omega_{c}} j \sigma\left(j \omega \mathbf{A}_{h}+\nabla \varphi_{h}\right) \overline{\nabla \hat{\varphi}} \\
& +\int_{\Omega_{c}} j \sigma\left(j \omega \mathbf{A}_{h}+\nabla \varphi_{h}\right) \overline{\nabla \Phi} \mid
\end{aligned}
$$


Introducing the three Clément interpolation operators $P_{C l}, I_{C l}$ and $I_{C l}^{0}$, we have $P_{C l} \mathbf{w} \in$ $X_{h}, I_{C l} \hat{\varphi} \in \Theta_{h}\left(\Omega_{c}\right)$ and $I_{C l}^{0} \Phi \in \Theta_{h}^{0}$. We define $\widetilde{I_{C l}^{0}}(\hat{\varphi})$ as an extension of $I_{C l} \hat{\varphi}$ in $\Omega$, such that $\widetilde{I_{C l}^{0}}(\hat{\varphi}) \in \Theta_{h}^{0}$. So, the orthogonality property from Lemma 2.7 leads to :

$$
\begin{aligned}
\omega\left|r\left(\mathbf{e}_{A}, e_{\varphi}\right)\right|= & \mid \int_{\Omega} \mathbf{J}_{s} \cdot \overline{-j\left(\mathbf{w}-P_{C l} \mathbf{w}\right)}-\int_{\Omega} \frac{1}{\mu} \operatorname{rot} \mathbf{A}_{h} \cdot \operatorname{rot} \overline{-j\left(\mathbf{w}-P_{C l} \mathbf{w}\right)} \\
& +\int_{\Omega_{c}} j \sigma\left(j \omega \mathbf{A}_{h}+\nabla \varphi_{h}\right) \overline{\left(\mathbf{w}-P_{C l} \mathbf{w}\right)} \\
& +\int_{\Omega_{c}} j \sigma\left(j \omega \mathbf{A}_{h}+\nabla \varphi_{h}\right) \overline{\nabla\left(\hat{\varphi}-\widetilde{I_{C l}^{0}} \hat{\varphi}\right)} \\
& +\int_{\Omega_{c}} j \sigma\left(j \omega \mathbf{A}_{h}+\nabla \varphi_{h}\right) \overline{\nabla\left(\Phi-I_{C l}^{0} \Phi\right)} \mid .
\end{aligned}
$$

Now, using the fact that $\mathbf{A}_{h} \in X(\Omega), \hat{\varphi} \in H_{0}^{1}(\Omega)$ and $\Phi \in H_{0}^{1}(\Omega)$ as well as Green's formula on each tetrahedron, we obtain:

$$
\begin{aligned}
\omega\left|r\left(\mathbf{e}_{A}, e_{\varphi}\right)\right|= & \mid \sum_{T \in \mathcal{T}}\left(\pi_{h} \mathbf{J}_{s}-\operatorname{rot}\left(\frac{1}{\mu} \operatorname{rot} \mathbf{A}_{h}\right)-\sigma\left(j \omega \mathbf{A}_{h}+\nabla \varphi_{h}\right),-j\left(\mathbf{w}-P_{C l} \mathbf{w}\right)\right)_{T} \\
& +\sum_{T \in \mathcal{T}}\left(\mathbf{J}_{s}-\pi_{h} \mathbf{J}_{s},-j\left(\mathbf{w}-P_{C l} \mathbf{w}\right)\right)_{T} \\
& +\sum_{F \in \mathcal{F}_{i n t}}\left(\left[\mathbf{n} \times \frac{1}{\mu} \operatorname{rot} \mathbf{A}_{h}\right]_{F},-j\left(\mathbf{w}-P_{C l} \mathbf{w}\right)\right)_{F} \\
& -\sum_{T \in \mathcal{T}}\left(\operatorname{div}\left(j \sigma\left(j \omega \mathbf{A}_{h}+\nabla \varphi_{h}\right)\right), \hat{\varphi}-\widetilde{I_{C l}^{0}} \hat{\varphi}\right)_{T} \\
& +\sum_{F \in \mathcal{F}_{\text {int }}}\left(\left[j \sigma\left(j \omega \mathbf{A}_{h}+\nabla \varphi_{h}\right) \cdot \mathbf{n}\right]_{F}, \hat{\varphi}-\widetilde{I_{C l}^{0}} \hat{\varphi}\right)_{F} \\
& -\sum_{T \in \mathcal{T}}\left(\operatorname{div}\left(j \sigma\left(j \omega \mathbf{A}_{h}+\nabla \varphi_{h}\right)\right), \Phi-I_{C l}^{0} \Phi\right)_{T} \\
& +\sum_{F \in \mathcal{F}_{\text {int }}}\left(\left[j \sigma\left(j \omega \mathbf{A}_{h}+\nabla \varphi_{h}\right) \cdot \mathbf{n}\right]_{F}, \Phi-I_{C l}^{0} \Phi\right)_{F} \mid
\end{aligned}
$$


The Cauchy-Schwarz inequality and the fact that $\sigma_{\mid \Omega_{e}}=0$ successively leads to :

$$
\begin{aligned}
& \omega\left|r\left(\mathbf{e}_{A}, e_{\varphi}\right)\right| \lesssim \sum_{T \in \mathcal{T}}\left\|\pi_{h} \mathbf{J}_{s}-\operatorname{rot}\left(\frac{1}{\mu} \operatorname{rot} \mathbf{A}_{h}\right)-\sigma\left(j \omega \mathbf{A}_{h}+\nabla \varphi_{h}\right)\right\|_{T}\left\|\mathbf{w}-P_{C l} \mathbf{w}\right\|_{T} \\
& +\sum_{T \in \mathcal{T}}\left\|\mathbf{J}_{s}-\pi_{h} \mathbf{J}_{s}\right\|_{T}\left\|\mathbf{w}-P_{C l} \mathbf{w}\right\|_{T} \\
& +\sum_{F \in \mathcal{F}_{\text {int }}}\left\|\left[\mathbf{n} \times \frac{1}{\mu} \operatorname{rot} \mathbf{A}_{h}\right]_{F}\right\|_{F}\left\|\mathbf{w}-P_{C l} \mathbf{w}\right\|_{F} \\
& +\sum_{T \in \mathcal{T}, T \in \Omega_{c}}\left\|\operatorname{div}\left(\sigma\left(j \omega \mathbf{A}_{h}+\nabla \varphi_{h}\right)\right)\right\|_{T}\left\|\hat{\varphi}-I_{C l} \hat{\varphi}\right\|_{T} \\
& +\sum_{F \in \mathcal{F}, F \in \overline{\Omega_{c}}}\left\|\left[\sigma\left(j \omega \mathbf{A}_{h}+\nabla \varphi_{h}\right) \cdot \mathbf{n}\right]_{F}\right\|_{F}\left\|\hat{\varphi}-I_{C l} \hat{\varphi}\right\|_{F} \\
& +\sum_{T \in \mathcal{T}}\left\|\operatorname{div}\left(\sigma\left(j \omega \mathbf{A}_{h}+\nabla \varphi_{h}\right)\right)\right\|_{T}\left\|\Phi-I_{C l}^{0} \Phi\right\|_{T} \\
& +\sum_{F \in \mathcal{F}_{\text {int }}}\left\|\left[\sigma\left(j \omega \mathbf{A}_{h}+\nabla \varphi_{h}\right) \cdot \mathbf{n}\right]_{F}\right\|_{F}\left\|\Phi-I_{C l}^{0}\right\|_{F} \\
& \omega\left|r\left(\mathbf{e}_{A}, e_{\varphi}\right)\right| \lesssim\left(\sum_{T \in \mathcal{T}} h_{T}^{2}\left\|\pi_{h} \mathbf{J}_{s}-\operatorname{rot}\left(\frac{1}{\mu} \operatorname{rot} \mathbf{A}_{h}\right)-\sigma\left(j \omega \mathbf{A}_{h}+\nabla \varphi_{h}\right)\right\|_{T}^{2}\right)^{\frac{1}{2}} \\
& \left(\sum_{T \in \mathcal{T}} h_{T}^{-2}\left\|\mathbf{w}-P_{C l} \mathbf{w}\right\|_{T}^{2}\right)^{\frac{1}{2}} \\
& +\left(\sum_{T \in \mathcal{T}} h_{T}^{2}\left\|\mathbf{J}_{s}-\pi_{h} \mathbf{J}_{s}\right\|_{T}^{2}\right)^{\frac{1}{2}}\left(\sum_{T \in \mathcal{T}} h_{T}^{-2}\left\|\mathbf{w}-P_{C l} \mathbf{w}\right\|_{T}^{2}\right)^{\frac{1}{2}} \\
& +\left(\sum_{F \in \mathcal{F}_{\text {int }}} h_{F}\left\|\left[\mathbf{n} \times \frac{1}{\mu} \operatorname{rot} \mathbf{A}_{h}\right]_{F}\right\|_{F}^{2}\right)^{\frac{1}{2}}\left(\sum_{F \in \mathcal{F}_{\text {int }}} h_{F}^{-1}\left\|\mathbf{w}-P_{C l} \mathbf{w}\right\|_{F}^{2}\right)^{\frac{1}{2}} \\
& +\left(\sum_{T \in \mathcal{T}, T \in \Omega_{c}} h_{T}^{2}\left\|\operatorname{div}\left(\sigma\left(j \omega \mathbf{A}_{h}+\nabla \varphi_{h}\right)\right)\right\|_{T}^{2}\right)^{\frac{1}{2}}\left(\sum_{T \in \mathcal{T}, T \in \Omega_{c}} h_{T}^{-2}\left\|\hat{\varphi}-I_{C l} \hat{\varphi}\right\|_{T}^{2}\right)^{\frac{1}{2}}
\end{aligned}
$$




$$
\begin{aligned}
& +\left(\sum_{F \in \mathcal{F}_{\text {int }}, F \in \overline{\Omega_{c}}} h_{F}\left\|\left[\sigma\left(j \omega \mathbf{A}_{h}+\nabla \varphi_{h}\right) \cdot \mathbf{n}\right]\right\|_{F}^{2}\right)^{\frac{1}{2}}\left(\sum_{F \in \mathcal{F}_{\text {int }}, F \in \overline{\Omega_{c}}} h_{F}^{-1}\left\|\hat{\varphi}-I_{C l} \hat{\varphi}\right\|_{F}^{2}\right)^{\frac{1}{2}} \\
& +\left(\sum_{T \in \mathcal{T}} h_{T}^{2}\left\|\operatorname{div}\left(\sigma\left(j \omega \mathbf{A}_{h}+\nabla \varphi_{h}\right)\right)\right\|_{T}^{2}\right)^{\frac{1}{2}}\left(\sum_{T \in \mathcal{T}} h_{T}^{-2}\left\|\Phi-I_{C l}^{0} \Phi\right\|_{T}^{2}\right)^{\frac{1}{2}} \\
& +\left(\sum_{F \in \mathcal{F}_{\text {int }}} h_{F}\left\|\left[\sigma\left(j \omega \mathbf{A}_{h}+\nabla \varphi_{h}\right) \cdot \mathbf{n}\right]_{F}\right\|_{F}^{2}\right)^{\frac{1}{2}}\left(\sum_{F \in \mathcal{F}_{\text {int }}} h_{F}^{-1}\left\|\Phi-I_{C l}^{0} \Phi\right\|_{F}^{2}\right)^{\frac{1}{2}}
\end{aligned}
$$

Now, we deduce from inequalities (37) to (42) that:

$$
\begin{aligned}
\omega\left|r\left(\mathbf{e}_{A}, e_{\varphi}\right)\right| \lesssim & \left(\sum_{T \in \mathcal{T}} h_{T}^{2}\left\|\pi_{h} \mathbf{J}_{s}-\operatorname{rot}\left(\frac{1}{\mu} \operatorname{rot} \mathbf{A}_{h}\right)-\sigma\left(j \omega \mathbf{A}_{h}+\nabla \varphi_{h}\right)\right\|_{T}^{2}\right)^{\frac{1}{2}}\left\|\nabla_{P} \mathbf{w}\right\| \\
& +\left(\sum_{T \in \mathcal{T}} h_{T}^{2}\left\|\mathbf{J}_{s}-\pi_{h} \mathbf{J}_{s}\right\|_{T}^{2}\right)^{\frac{1}{2}}\left\|\nabla_{P} \mathbf{w}\right\| \\
& +\left(\sum_{F \in \mathcal{F}_{\text {int }}} h_{F}\left\|\left[\mathbf{n} \times \frac{1}{\mu} \operatorname{rot} \mathbf{A}_{h}\right]_{F}\right\|_{F}^{2}\right)^{\frac{1}{2}}\left\|\nabla_{P} \mathbf{w}\right\| \\
& +\left(\sum_{T \in \mathcal{T}, T \in \Omega_{c}} h_{T}^{2}\left\|\operatorname{div}\left(\sigma\left(j \omega \mathbf{A}_{h}+\nabla \varphi_{h}\right)\right)\right\|_{T}^{2}\right)^{\frac{1}{2}}|\hat{\varphi}|_{1, \Omega_{c}} \\
& +\left(\sum_{F \in \mathcal{F}_{\text {int }}, F \in \bar{\Omega}_{c}} h_{F}\left\|\left[\sigma\left(j \omega \mathbf{A}_{h}+\nabla \varphi_{h}\right) \cdot \mathbf{n}\right]_{F}\right\|_{F}^{2}\right)^{\frac{1}{2}}|\hat{\varphi}|_{1, \Omega_{c}} \\
& +\left(\sum_{T \in \mathcal{T}} h_{T}^{2}\left\|\operatorname{div}\left(\sigma\left(j \omega \mathbf{A}_{h}+\nabla \varphi_{h}\right)\right)\right\|_{T}^{2}\right)^{\frac{1}{2}}|\Phi|_{1} \\
& +\left(\sum_{F \in \mathcal{F}_{\text {int }}} h_{F}\left\|\left[\sigma\left(j \omega \mathbf{A}_{h}+\nabla \varphi_{h}\right) \cdot \mathbf{n}\right]\right\|_{F}^{2}\right)^{\frac{1}{2}}|\Phi|_{1} .
\end{aligned}
$$


Let us recall from Theorem 3.1 that (25), (26), (28) and (29), lead to :

$$
\begin{aligned}
|\hat{\varphi}|_{1, \Omega_{c}} & \lesssim\left\|j \omega \mathbf{e}_{A}+\nabla e_{\varphi}\right\|_{\Omega_{c}} \\
|\Phi|_{1} & \lesssim\left\|j \omega \mathbf{e}_{A}+\nabla e_{\varphi}\right\|_{\Omega_{c}}+\omega\left\|\operatorname{rot} \mathbf{e}_{A}\right\|, \\
\left\|\nabla_{P} \mathbf{w}\right\| & \lesssim\left\|j \omega \mathbf{e}_{A}+\nabla e_{\varphi}\right\|_{\Omega_{c}}+\omega\left\|\operatorname{rot} \mathbf{e}_{A}\right\|,
\end{aligned}
$$

Moreover,

$$
\left\|j \omega \mathbf{e}_{A}+\nabla e_{\varphi}\right\|_{\Omega_{c}} \leq \omega^{1 / 2} \max _{T \in \Omega_{c}}\left(\frac{1}{\sigma_{T}^{1 / 2}}\right)\left\|\frac{\sigma^{1 / 2}}{\omega^{1 / 2}}\left(j \omega \mathbf{e}_{A}+\nabla e_{\varphi}\right)\right\|_{\Omega_{c}},
$$

and

$$
\left\|\operatorname{rot} \mathbf{e}_{A}\right\|_{\Omega} \lesssim \max _{T \in \Omega}\left(\mu_{T}^{1 / 2}\right)\left\|\sqrt{\frac{1}{\mu}} \operatorname{rot} \mathbf{e}_{A}\right\|
$$

Hence we have :

$$
\begin{aligned}
& |\Phi|_{1} \lesssim \max \left\{\omega \max _{T \in \Omega}\left(\mu_{T}^{1 / 2}\right), \omega^{1 / 2} \max _{T \in \Omega_{c}}\left(\frac{1}{\sigma_{T}^{1 / 2}}\right)\right\}\left(\left\|\sqrt{\frac{1}{\mu}} \operatorname{rot} \mathbf{e}_{A}\right\|+\left\|\frac{\sigma^{1 / 2}}{\omega^{1 / 2}}\left(j \omega \mathbf{e}_{A}+\nabla e_{\varphi}\right)\right\|_{\Omega_{c}}\right), \\
& \left\|\nabla_{P} \mathbf{w}\right\| \lesssim \max \left\{\omega \max _{T \in \Omega}\left(\mu_{T}^{1 / 2}\right), \omega^{1 / 2} \max _{T \in \Omega_{c}}\left(\frac{1}{\sigma_{T}^{1 / 2}}\right)\right\}\left(\left\|\sqrt{\frac{1}{\mu}} \operatorname{rot} \mathbf{e}_{A}\right\|+\left\|\frac{\sigma^{1 / 2}}{\omega^{1 / 2}}\left(j \omega \mathbf{e}_{A}+\nabla e_{\varphi}\right)\right\|_{\Omega_{c}}\right) .
\end{aligned}
$$

Consequently, using (48) and these last estimates in (52) we derive the reliability of our estimator, namely the estimate (51).

\subsection{Efficiency}

Now, in order to derive the efficiency of our estimator, we have to bound each part of the estimator by the local error.

Lemma 4.7 We have :

$$
\eta_{T ; 1} \lesssim \frac{1}{\mu_{T}^{1 / 2}}\left\|\sqrt{\frac{1}{\mu}} \operatorname{rot} \mathbf{e}_{\mathbf{A}}\right\|_{T}+h_{T} \omega^{1 / 2} \sigma_{T}^{1 / 2}\left\|\frac{\sigma^{1 / 2}}{\omega^{1 / 2}}\left(j \omega \mathbf{e}_{\mathbf{A}}+\nabla e_{\varphi}\right)\right\|_{T}+\eta_{T ; 2}
$$

Proof: Let us define $\mathbf{j}_{h}$ on $T$ by :

$$
\mathbf{j}_{h}=\pi_{h} \mathbf{J}_{s}-\operatorname{rot}\left(\frac{1}{\mu} \operatorname{rot} \mathbf{A}_{h}\right)-\sigma\left(j \omega \mathbf{A}_{h}+\nabla \varphi_{h}\right)
$$

By the definition of $\eta_{T ; 1}$, we have :

$$
\eta_{T ; 1}^{2}=h_{T}^{2}\left\|\mathbf{j}_{h}\right\|_{T}^{2}
$$


and from the inverse inequality (43) as well as from the Green formula, we get :

$$
\begin{aligned}
\frac{\eta_{T ; 1}^{2}}{h_{T}^{2}} \lesssim & \left\|\left(\mathbf{J}_{s}-\pi_{h} \mathbf{J}_{s}\right)\right\|_{T}\left\|b_{T} \mathbf{j}_{h}\right\|_{T} \\
& +\int_{T} \mathbf{J}_{s} \overline{b_{T} \mathbf{j}_{h}}-\int_{T} \frac{1}{\mu} \operatorname{rot} \mathbf{A}_{h} \overline{\operatorname{rot} b_{T} \mathbf{j}_{h}} \\
& -\int_{T} \sigma\left(j \omega \mathbf{A}_{h}+\nabla \varphi_{h}\right) \overline{b_{T} \mathbf{j}_{h}}
\end{aligned}
$$

Since $b_{T} \mathbf{j}_{h} \in X(\Omega)$, Lemma 2.3 leads to :

$$
\int_{\Omega} \frac{1}{\mu} \operatorname{rot} \mathbf{A} \operatorname{rot} \overline{b_{T} \mathbf{j}_{h}}-\int_{\Omega_{c}} \frac{j \sigma}{\omega}(j \omega \mathbf{A}+\nabla \varphi)\left(\overline{j \omega b_{T} \mathbf{j}_{h}}\right)=\int_{\Omega} \mathbf{J}_{s} \overline{b_{T} \mathbf{j}_{h}}
$$

So that :

$$
\begin{aligned}
& \int_{T} \mathbf{J}_{s} \overline{b_{T} \mathbf{j}_{h}}-\int_{T} \frac{1}{\mu} \operatorname{rot} \mathbf{A}_{h} \operatorname{rot} \overline{b_{T} \dot{\mathbf{j}}_{h}}+\int_{\Omega_{c}} \frac{j \sigma}{\omega}\left(j \omega \mathbf{A}_{h}+\nabla \varphi_{h}\right)\left(\overline{j \omega b_{T} \mathbf{j}_{h}}\right) \\
= & \int_{T} \frac{1}{\mu} \operatorname{rot} \mathbf{e}_{\mathbf{A}} \operatorname{rot} \overline{b_{T} \mathbf{j}_{h}}-\int_{T} \frac{j \sigma}{\omega}\left(j \omega \mathbf{e}_{\mathbf{A}}+\nabla e_{\varphi}\right)\left(\overline{j \omega b_{T} \mathbf{j}_{h}}\right)
\end{aligned}
$$

From the Cauchy-Schwarz inequality, we obtain :

$$
\begin{aligned}
& \int_{T} \mathbf{J}_{s} \overline{b_{T} \mathbf{j}_{h}}-\int_{T} \frac{1}{\mu} \operatorname{rot} \mathbf{A}_{h} \operatorname{rot} \overline{b_{T} \mathbf{j}_{h}}+\int_{T} \frac{j \sigma}{\omega}\left(j \omega \mathbf{A}_{h}+\nabla \varphi_{h}\right)\left(\overline{j \omega b_{T} \mathbf{j}_{h}}\right) \\
\lesssim & \left\|\sqrt{\frac{1}{\mu}} \operatorname{rot} \mathbf{e}_{\mathbf{A}}\right\|_{T}\left\|\sqrt{\frac{1}{\mu}} \operatorname{rot} b_{T} \mathbf{j}_{h}\right\|_{T}+\left\|\sigma\left(j \omega \mathbf{e}_{\mathbf{A}}+\nabla e_{\varphi}\right)\right\|_{T}\left\|b_{T} \mathbf{j}_{h}\right\|_{L^{2}(K)} \\
\lesssim & \left\|\sqrt{\frac{1}{\mu}} \operatorname{rot} \mathbf{e}_{\mathbf{A}}\right\|\left\|_{T}\right\| \sqrt{\frac{1}{\mu}} \operatorname{rot} b_{T} \mathbf{j}_{h}\left\|_{T}+\omega^{1 / 2} \sigma_{T}^{1 / 2}\right\| \frac{\sigma^{1 / 2}}{\omega^{1 / 2}}\left(j \omega \mathbf{e}_{\mathbf{A}}+\nabla e_{\varphi}\right)\left\|_{T}\right\| b_{T} \mathbf{j}_{h} \|_{L^{2}(K)}
\end{aligned}
$$

From the inverse inequality (44), we have :

$$
\left\|\operatorname{rot} b_{T} \mathbf{j}_{h}\right\|_{T} \leq\left\|\nabla\left(b_{T} \mathbf{j}_{h}\right)\right\|_{T} \lesssim h_{T}^{-1}\left\|\mathbf{j}_{h}\right\|_{T}
$$

From (54) to (56) we deduce :

$$
\frac{\eta_{T ; 1}^{2}}{h_{T}^{2}} \lesssim\left(\left\|\left(\mathbf{J}_{s}-\pi_{h} \mathbf{J}_{s}\right)\right\|_{T}+\frac{1}{\mu_{T}^{1 / 2}} h_{T}^{-1}\left\|\sqrt{\frac{1}{\mu}} \operatorname{rot} \mathbf{e}_{\mathbf{A}}\right\|_{T}+\omega^{1 / 2} \sigma_{T}^{1 / 2}\left\|\frac{\sigma^{1 / 2}}{\omega^{1 / 2}}\left(j \omega \mathbf{e}_{\mathbf{A}}+\nabla e_{\varphi}\right)\right\|_{T}\right)\left\|\mathbf{j}_{h}\right\|_{T}
$$

which proves $(53)$. 
Remark 4.8 Like already mentioned in remark $4.4, \eta_{T ; 2}$ is superconvergent and we should have directly written :

$$
\eta_{T ; 1} \lesssim \frac{1}{\mu_{T}^{1 / 2}}\left\|\sqrt{\frac{1}{\mu}} \operatorname{rot} \mathbf{e}_{\mathbf{A}}\right\|_{T}+h_{T} \omega^{1 / 2} \sigma_{T}^{1 / 2}\left\|\frac{\sigma^{1 / 2}}{\omega^{1 / 2}}\left(j \omega \mathbf{e}_{\mathbf{A}}+\nabla e_{\varphi}\right)\right\|_{T}+\text { h.o.t }
$$

where h.o.t. corresponds to a higher order term.

Lemma 4.9 Let $F$ be a common face of the tetrahedra $T_{1}$ and $T_{2}$, then we have :

$$
\begin{aligned}
\eta_{F ; 1} \lesssim & \frac{1}{\mu_{F, \min }^{1 / 2}}\left\|\sqrt{\frac{1}{\mu}} \operatorname{rot} \mathbf{e}_{\mathbf{A}}\right\|_{T_{1} \cup T_{2}}+h_{F} \omega^{1 / 2} \sigma_{F, \max }^{1 / 2}\left\|\frac{\sigma^{1 / 2}}{\omega^{1 / 2}}\left(j \omega \mathbf{e}_{\mathbf{A}}+\nabla e_{\varphi}\right)\right\|_{T_{1} \cup T_{2}} \\
& +\eta_{T_{1} ; 2}+\eta_{T_{2} ; 2}+\eta_{T_{1} ; 1}+\eta_{T_{2} ; 1}
\end{aligned}
$$

where we have set

$$
\sigma_{F, \max }=\max \left(\sigma_{T_{1}}, \sigma_{T_{2}}\right) \text { and } \mu_{F, \text { min }}=\min \left(\mu_{T_{1}}, \mu_{T_{2}}\right)
$$

Proof: Let us here define $F \mathbf{j}_{h}=\left[\mathbf{n} \times \frac{1}{\mu} \operatorname{rot} \mathbf{A}_{h}\right]_{F}$. From (45) we have:

$$
\frac{\eta_{F ; 1}^{2}}{h_{F}}=\left\|\mathbf{j}_{h}\right\|_{F}^{2} \lesssim \int_{F}\left[\mathbf{n} \times \frac{1}{\mu} \operatorname{rot} \mathbf{A}_{h}\right]_{F} \overline{b_{F} \mathbf{j}_{h}}
$$

If we introduce $w_{F}=\mathrm{F}_{\text {ext }}\left(\mathbf{j}_{h}\right) b_{F} \in H_{0}^{1}\left(T_{1} \cup T_{2}\right)$, using Green formula, we get :

$$
\begin{aligned}
\frac{\eta_{F ; 1}^{2}}{h_{F}} \lesssim & \sum_{i=1}^{2} \int_{T_{i}}-\operatorname{rot}\left(\frac{1}{\mu} \operatorname{rot} \mathbf{A}_{h}\right) \overline{w_{F}}+\frac{1}{\mu} \operatorname{rot} \mathbf{A}_{h} \operatorname{rot} \overline{w_{F}} \\
= & \sum_{i=1}^{2} \int_{T_{i}}\left(\mathbf{J}_{s}-\operatorname{rot}\left(\frac{1}{\mu} \operatorname{rot} \mathbf{A}_{h}\right)-\sigma\left(j \omega \mathbf{A}_{h}+\nabla \varphi_{h}\right)\right) \overline{w_{F}} \\
& -\int_{T_{1} \cup T_{2}} \mathbf{J}_{s} \overline{w_{F}}-\frac{1}{\mu} \operatorname{rot} \mathbf{A}_{h} \operatorname{rot} \overline{w_{F}}-\sigma\left(j \omega \mathbf{A}_{h}+\nabla \varphi_{h}\right) \overline{w_{F}}
\end{aligned}
$$

so that :

$$
\begin{aligned}
\frac{\eta_{F ; 1}^{2}}{h_{F}} \lesssim & \sum_{i=1}^{2} \int_{T_{i}}\left\|\mathbf{J}_{s}-\operatorname{rot}\left(\frac{1}{\mu} \operatorname{rot} \mathbf{A}_{h}\right)-\sigma\left(j \omega \mathbf{A}_{h}+\nabla \varphi_{h}\right)\right\|_{T_{i}}\left\|w_{F}\right\|_{T_{i}} \\
& +\left\|\sqrt{\frac{1}{\mu}} \operatorname{rot} \mathbf{e}_{\mathbf{A}}\right\|_{\left.T_{1} \cup T_{2}\right)}\left\|\frac{1}{\mu^{1 / 2}} \operatorname{rot} w_{F}\right\|_{T_{1} \cup T_{2}} \\
& +\left\|\sigma\left(j \omega \mathbf{e}_{\mathbf{A}}+\nabla e_{\varphi}\right)\right\|_{T_{1} \cup T_{2}}\left\|w_{F}\right\|_{T_{1} \cup T_{2}}
\end{aligned}
$$


Now, using the inverse inequalities (46) and (47) and using the quantities $\mu_{F, \min }=\min \left(\mu_{T_{1}}, \mu_{T_{2}}\right)$ and $\sigma_{F, \max }=\max \left(\sigma_{T_{1}}, \sigma_{T_{2}}\right)$, we get :

$$
\begin{aligned}
\frac{\eta_{F ; 1}^{2}}{h_{F}} \lesssim & h_{F}^{1 / 2}\left(h_{T_{1}}^{-1} \eta_{T_{1} ; 2}+h_{T_{2}}^{-1} \eta_{T_{2} ; 2}+h_{T_{1}}^{-1} \eta_{T_{1} ; 1}+h_{T_{2}}^{-1} \eta_{T_{2} ; 1}\right)\left\|\mathbf{j}_{h}\right\|_{F} \\
& +\frac{h_{F}^{-1 / 2}}{\mu_{F, \min }^{1 / 2}}\left\|\sqrt{\frac{1}{\mu}} \operatorname{rot} \mathbf{e}_{\mathbf{A}}\right\|_{T_{1} \cup T_{2}}\left\|\mathbf{j}_{h}\right\|_{F} \\
& +h_{F}^{1 / 2} \omega^{1 / 2} \sigma_{F, \max }^{1 / 2}\left\|\frac{\sigma^{1 / 2}}{\omega^{1 / 2}}\left(j \omega \mathbf{e}_{\mathbf{A}}+\nabla e_{\varphi}\right)\right\|_{T_{1} \cup T_{2}}\left\|\mathbf{j}_{h}\right\|_{F} .
\end{aligned}
$$

So we conclude :

$$
\begin{aligned}
\eta_{F ; 1} \lesssim & h_{F}\left(h_{T_{1}}^{-1} \eta_{T_{1} ; 2}+h_{T_{2}}^{-1} \eta_{T_{2} ; 2}+h_{T_{1}}^{-1} \eta_{T_{1} ; 1}+h_{T_{2}}^{-1} \eta_{T_{2} ; 1}\right) \\
& +\frac{1}{\mu_{F, \min }^{1 / 2}}\left\|\sqrt{\frac{1}{\mu}} \operatorname{rot} \mathbf{e}_{\mathbf{A}}\right\|_{T_{1} \cup T_{2}}+h_{F} \omega^{1 / 2} \sigma_{F, \max }^{1 / 2}\left\|\frac{\sigma^{1 / 2}}{\omega^{1 / 2}}\left(j \omega \mathbf{e}_{\mathbf{A}}+\nabla e_{\varphi}\right)\right\|_{T_{1} \cup T_{2}}
\end{aligned}
$$

Since the mesh is regular, we have obtained (57).

Remark 4.10 Once again, invoking the superconvergence property of $\eta_{T_{1} ; 2}$ and $\eta_{T_{2} ; 2}$, we should have written :

$$
\eta_{F ; 1} \lesssim \frac{1}{\mu_{F, \min }^{1 / 2}}\left\|\frac{1}{\mu^{1 / 2}} \operatorname{rot} \mathbf{e}_{\mathbf{A}}\right\|_{T_{1} \cup T_{2}}+h_{F} \omega^{1 / 2} \sigma_{F, \max }^{1 / 2}\left\|\frac{\sigma^{1 / 2}}{\omega^{1 / 2}}\left(j \omega \mathbf{e}_{\mathbf{A}}+\nabla e_{\varphi}\right)\right\|_{T_{1} \cup T_{2}}+\eta_{T_{1} ; 1}+\eta_{T_{2} ; 1}+\text { h.o.t. }
$$

Lemma 4.11 Let $F$ be a common face of the tetrahedra $T_{1}$ and $T_{2}$, then we have :

$$
\eta_{F ; 2} \lesssim \omega^{1 / 2} \sigma_{F, \max }^{1 / 2}\left\|\frac{\sigma^{1 / 2}}{\omega^{1 / 2}}\left(j \omega \mathbf{e}_{\mathbf{A}}+\nabla e_{\varphi}\right)\right\|_{T_{1} \cup T_{2}}
$$

Proof: This time we define $\mathbf{j}_{h}=\left[\sigma\left(j \omega \mathbf{A}_{h}+\nabla \varphi_{h}\right) \cdot \mathbf{n}\right]_{F}$. From (45) we have:

$$
\frac{\eta_{F ; 2}^{2}}{h_{F}}=\left\|\mathbf{j}_{h}\right\|_{F}^{2} \lesssim \int_{F}\left[\sigma\left(j \omega \mathbf{A}_{h}+\nabla \varphi_{h}\right) \cdot \mathbf{n}\right]_{F} \overline{b_{F} \mathbf{j}_{h}}
$$


If we introduce $w_{F}=\mathrm{F}_{\text {ext }}\left(\mathbf{j}_{h}\right) b_{F} \in H_{0}^{1}\left(T_{1} \cup T_{2}\right)$, using Green formula, we get :

$$
\begin{aligned}
\frac{\eta_{F ; 2}^{2}}{h_{F}} \lesssim & \sum_{i=1}^{2} \int_{T_{i}}-\sigma\left(j \omega \mathbf{A}_{h}+\nabla \varphi_{h}\right) \overline{\nabla w_{F}}+\operatorname{div}\left(\sigma\left(j \omega \mathbf{A}_{h}+\nabla \varphi_{h}\right)\right) \overline{w_{F}} \\
= & \int_{T_{1} \cup T_{2}}\left(\sigma\left(j \omega \mathbf{A}+\nabla \varphi_{h}\right)-\sigma\left(j \omega \mathbf{A}_{h}+\nabla \varphi_{h}\right)\right) \overline{\nabla w_{F}} \\
& +\sum_{i=1}^{2} \int_{T_{i}} \operatorname{div}\left(\sigma\left(j \omega \mathbf{A}_{h}+\nabla \varphi_{h}\right)\right) \overline{w_{F}}
\end{aligned}
$$

so that :

$$
\begin{aligned}
\frac{\eta_{F ; 2}^{2}}{h_{F}} \lesssim & \sum_{i=1}^{2} \int_{T_{i}}\left\|\operatorname{div}\left(\sigma\left(j \omega \mathbf{A}_{h}+\nabla \varphi_{h}\right)\right)\right\|_{T_{i}}\left\|w_{F}\right\|_{T_{i}} \\
& +\left\|\frac{\sigma^{1 / 2}}{\omega^{1 / 2}}\left(j \omega \mathbf{e}_{\mathbf{A}}+\nabla e_{\varphi}\right)\right\|_{T_{1} \cup T_{2}}\left\|\omega^{1 / 2} \sigma^{1 / 2} \nabla w_{F}\right\|_{T_{1} \cup T_{2}}
\end{aligned}
$$

Now, using the inverse inequalities (47), the definition of $\sigma_{F, \max }=\max \left(\sigma_{T_{1}}, \sigma_{T_{2}}\right)$, and recalling that $\eta_{T ; 3}=0$, we get :

$$
\frac{\eta_{F ; 2}^{2}}{h_{F}} \lesssim\left\|\frac{\sigma^{1 / 2}}{\omega^{1 / 2}}\left(j \omega \mathbf{e}_{\mathbf{A}}+\nabla e_{\varphi}\right)\right\|_{T_{1} \cup T_{2}} \omega^{1 / 2} \sigma_{F, \max }^{1 / 2} h_{F}^{-1 / 2}\left\|\mathbf{j}_{h}\right\|_{F}
$$

This proves (62).

Theorem 4.12 We have the efficiency of our estimator:

$$
\eta_{T} \lesssim C_{T, \text { down }}\left(\left\|\sqrt{\frac{1}{\mu}} \operatorname{rot} \mathbf{e}_{\mathbf{A}}\right\|_{T_{1} \cup T_{2}}+\left\|\frac{\sigma^{1 / 2}}{\omega^{1 / 2}}\left(j \omega \mathbf{e}_{\mathbf{A}}+\nabla e_{\varphi}\right)\right\|_{T_{1} \cup T_{2}}\right)^{1 / 2}+\text { h.o.t. }
$$

with

$$
C_{T, \text { down }}=\max _{\bar{T}^{\prime} \cap \bar{T} \neq \emptyset} \max \left\{\mu_{T^{\prime}}^{-1 / 2}, \omega^{1 / 2} h_{T^{\prime}} \sigma_{T^{\prime}}^{1 / 2}\right\}
$$

Proof: Direct consequence of Lemmas 4.7, 4.9 and 4.11.

\section{Numerical validation}

In this section, some numerical experiments are performed to underline and confirm our theoretical predictions with the use of the software CARMEL 3D [10]. The first one (section 5.1) corresponds to an analytical test, for which the data have been built to have in hand 
an exact solution, in order to compare the estimator to the exact error. The second one (section 5.2) is a classical physical benchmark test involving strong singularities, for which no analytical solution is available, but which allows to show the behavior of the estimator in real situations.

\subsection{Analytical test}

This analytical test consists in solving the discrete formulation (21) on the domain $\Omega=$ $[-2,5] \times[-2,2] \times[-2,2]$, where $\Omega_{c}=[2,4] \times[-1,1] \times[-1,1]$, with $\mu \equiv 1$ on $\Omega, \sigma \equiv 1$ on $\Omega_{c}$ and $\omega=2 \pi$. First of all, define $f$ on $\Omega$ by :

$$
f(x, y, z)=\left\{\begin{array}{lll}
\left(x^{2}-1\right)^{4}\left(y^{2}-1\right)^{4}\left(z^{2}-1\right)^{4} & \text { in } \quad \Omega_{J}=[-1,1]^{3} \\
0 & \text { in } \quad \Omega \backslash \Omega_{J}
\end{array}\right.
$$

where $\Omega_{c} \cap \Omega_{J}=\emptyset$. It allows to define $A$ on $\Omega$ by :

$$
\mathbf{A}=\operatorname{rot}\left(\begin{array}{l}
f \\
0 \\
0
\end{array}\right)
$$

and we take $\varphi \equiv 0$ in $\Omega_{c}$. It is then possible from (9) to derive the value of $\mathbf{J}_{s}$ corresponding to the exact solution $(\mathbf{A}, 0)$. Let us note that from this choice and because of the regularity of $f$, we have $\operatorname{div} \mathbf{J}_{s}=0$ in $\Omega$.

To begin with, we check that the numerical solution converges towards the exact one. We plot on Figure 2 the error $\left(\int_{\Omega} \frac{1}{\mu}\left|\operatorname{rot} \mathbf{e}_{A}\right|^{2}+\int_{\Omega_{c}} \frac{\sigma}{\omega}\left|j \omega \mathbf{e}_{A}+\nabla e_{\varphi}\right|^{2}\right)^{1 / 2}$ as a function of the total number of degrees of freedom $D o F$. As we can see, the order of convergence is equal to $-1 / 3$ in $D o F$, corresponding to the order 1 in $h$ for the three-dimensional case since regular meshes are used. It shows a good behavior compared to expected theoretically results.

Now we investigate the theoretical results about the upper error bound (Theorem 4.6). We consider the ratio Error/ $\eta$ as a function of $D o F$. This ratio, the so-called effectivity index, is related to the global upper error bound and measures the reliability of the estimator. We see in Figure 3 that this effectivity index is bounded by a constant around 0.13 which is quite reasonable.

At last, we compare two error maps in the plane $z=0$. The first one (Figure 4(a)) corresponds to the exact error distribution, computed with the knowledge of the analytical solution. The second one (Figure $4(\mathrm{~b})$ ) is generated by using the values of the computed local estimators. It is clear that the estimators provide an error map in very good accordance with the actual error distribution. Consequently, it should be successively used to drive an adaptive mesh-refinement strategy. 


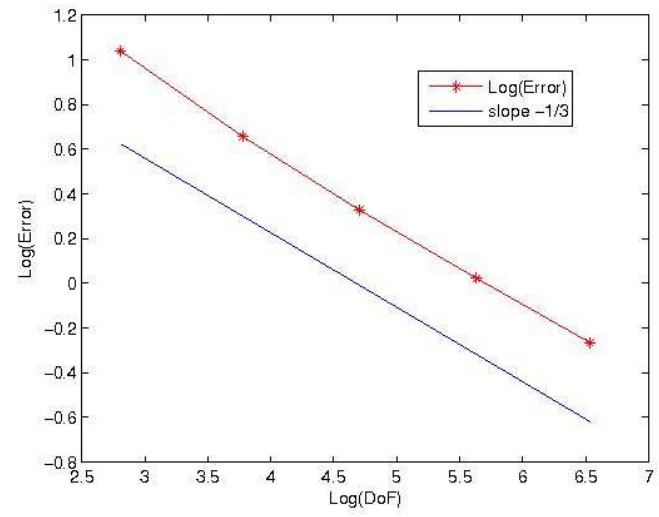

Figure 2: Exact error in its dependence on DoF.

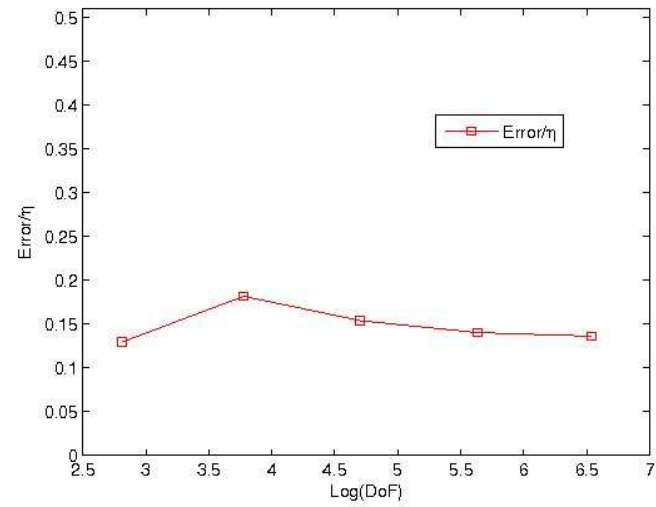

Figure 3: $\frac{\text { Error }}{\eta}$ in its dependence on $D o F$.

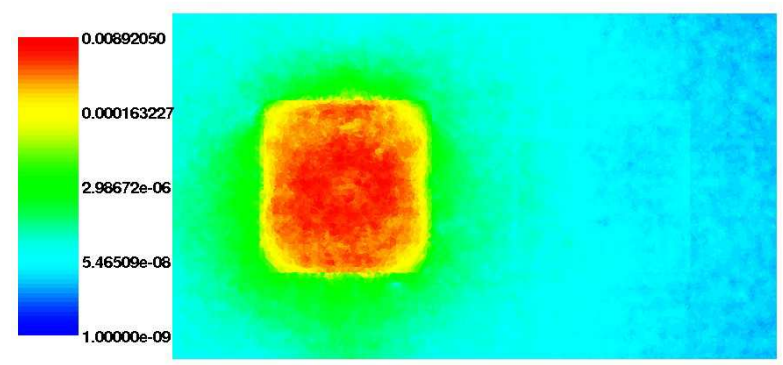

(a) Exact error.

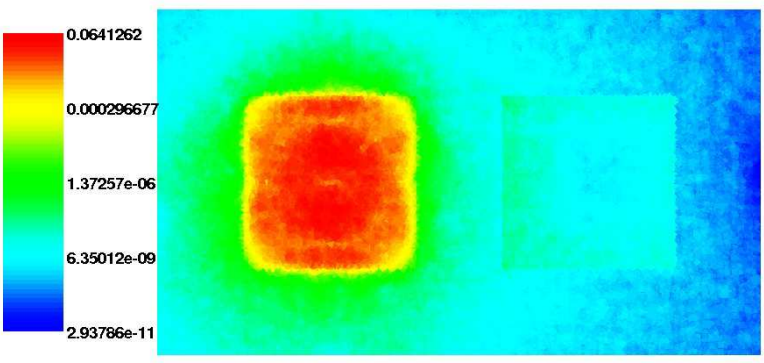

(b) Estimator.

Figure 4: Local Error Maps in the plane $z=0$. 


\subsection{Physical benchmark test}

In order to extend the analysis of the estimator, the problem 7 of Team Workshop has been modeled [11]. The computation model consists in an asymmetrical conductor with one hole and an exciting coil like displayed in Figure 5. Here we have $\mu=4 \pi 10^{-7}$ (corresponding to the air magnetic permeability in $\mathrm{H} / \mathrm{m}$ ) and $\sigma=3.62510^{+7}$ (corresponding to the aluminium electrical conductivity in $\mathrm{S} / \mathrm{m}$ ).
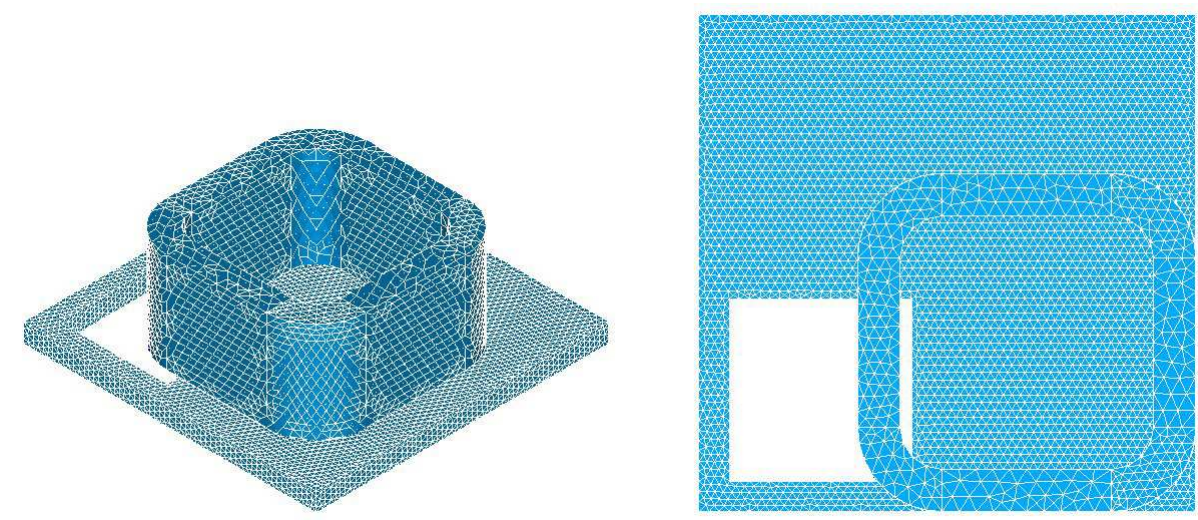

Figure 5: Domain and Mesh of Problem 7.

The value of $\mathbf{J}_{s}$ in the coil is imposed, and we consider several values of the frequency $f=2 \pi / \omega$ in order to make the induced current $\mathbf{J}_{e}=\sigma(j \omega \mathbf{A}+\nabla \varphi)$ varying in $\Omega_{c}$. We are interested in the Joule heating value $H$ and the magnetic energy $E$, respectively given by :

$$
\begin{gathered}
H=\int_{\Omega_{c}} \sigma|j \omega \mathbf{A}+\nabla \varphi|^{2}, \\
E=\int_{\Omega} \frac{1}{\mu}|\operatorname{rot} \mathbf{A}|^{2} .
\end{gathered}
$$

As physically expected, Table 1 shows that the larger the frequency is, the larger the Joule heating value is, whereas the magnetic energy remains the same. Table 2 shows for each frequency the values of each part of the computed error estimator, namely :

$$
\eta_{1}=\sum_{T \in \mathcal{T}} \eta_{T ; 1}^{2}, \quad \eta_{1}^{(F)}=\sum_{T \in \mathcal{T}} \eta_{F ; 1}^{2}, \quad \eta_{2}^{(F)}=\sum_{T \in \mathcal{T}} \eta_{F ; 2}^{2} .
$$

While the contributions $\eta_{1}$ and $\eta_{1}^{(F)}$ remain nearly unchanged, the values of $\eta_{2}^{(F)}$ are increasing with the frequency. Indeed, it is well-known that for this benchmark the numerical error is mainly due to the jump of the normal component of $\mathbf{J}_{\mathbf{e}}$ between the elements and that the higher the frequency is, the larger this error is. So the estimator behavior is very close to the expected error one. 
Figure 6(a) shows the induced current $\mathbf{J}_{e}$ computed in the conductor and Figure 6(b) the current vector field around the top right corner of the hole at the frequency $f=200 \mathrm{~Hz}$. Local estimator maps in the coil and in the same plane as in Figure 6(a) are respectively displayed in Figures 7(a) and 7(b). The error in the coil is concentrated in the corners. As expected the error estimators indicate that the largest errors are located where eddy currents are large and around the hole. Once again, it should be succefully used in order to drive a local mesh refinement strategy.

\begin{tabular}{|c|c|c|}
\hline Frequency $(\mathrm{Hz})$ & Joule Heating $\mathrm{H}(\mathrm{W})$ & Magnetic Energy E $(\mathrm{J})$ \\
\hline 200 & 18.603 & 1.1098 \\
2000 & 61.464 & 1.0979 \\
20000 & 116.10 & 1.0953 \\
200000 & 133.33 & 1.0951 \\
\hline
\end{tabular}

Table 1: Joule Heating and Magnetic Energy with different frequencies.

\begin{tabular}{|c|c|c|c|c|}
\hline Frequency $(\mathrm{Hz})$ & $\eta_{1}$ & $\eta_{1}^{(F)}$ & $\eta_{2}^{(F)}$ & $\eta$ \\
\hline 200 & $6.4644 \mathrm{E}+02$ & $4.6697 \mathrm{E}+02$ & $3.0531 \mathrm{E}+04$ & $3.0542 \mathrm{E}+04$ \\
2000 & $6.9607 \mathrm{E}+02$ & $4.7653 \mathrm{E}+02$ & $1.0191 \mathrm{E}+05$ & $1.0191 \mathrm{E}+05$ \\
20000 & $7.4859 \mathrm{E}+02$ & $4.7938 \mathrm{E}+02$ & $1.8083 \mathrm{E}+05$ & $1.8083 \mathrm{E}+05$ \\
200000 & $7.6401 \mathrm{E}+02$ & $4.7978 \mathrm{E}+02$ & $2.0081 \mathrm{E}+05$ & $2.0081 \mathrm{E}+05$ \\
\hline
\end{tabular}

Table 2: Estimator with different frequencies.

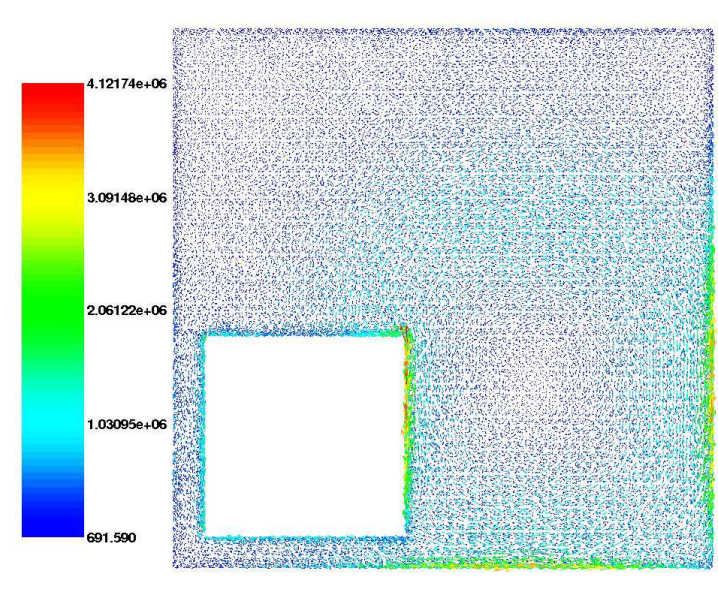

(a) Induced current.

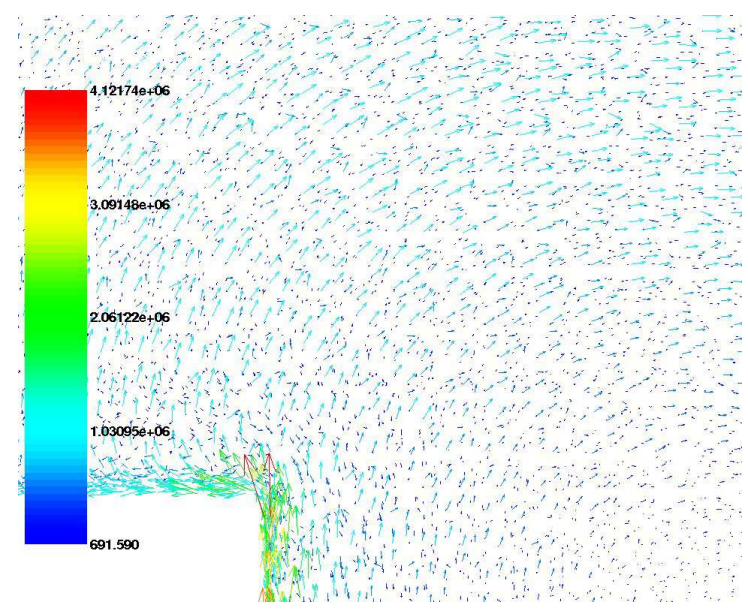

(b) Zoom in the corner.

Figure 6: View of the induced current at $200 \mathrm{~Hz}$. 


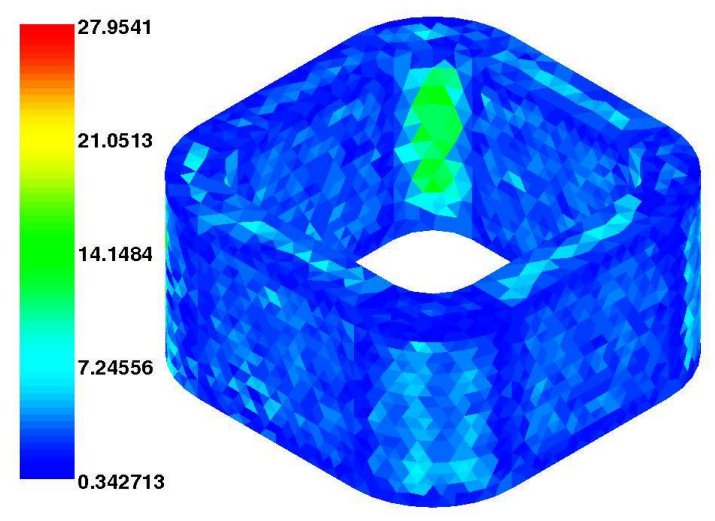

(a) Estimator in the coil.

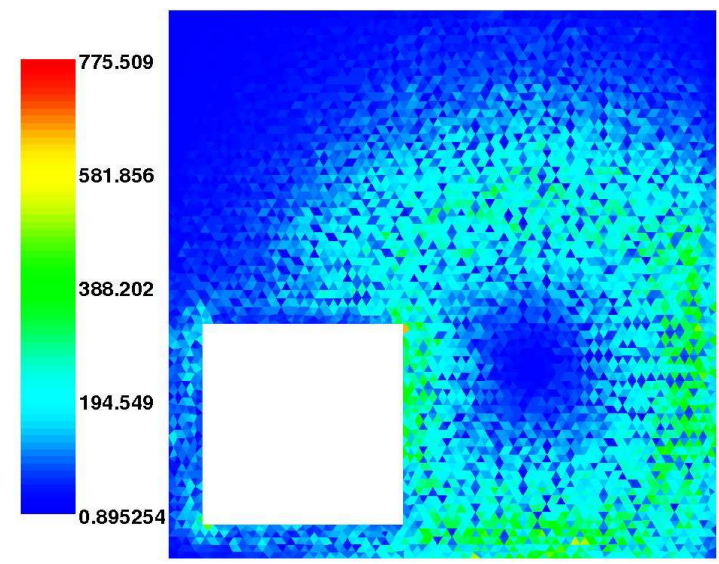

(b) Estimator in the conductor.

Figure 7: Local Estimator Map at $200 \mathrm{~Hz}$.

\section{References}

[1] M. Ainsworth and J.T. Oden. A posteriori error estimation in finite element analysis. Pure and Applied Mathematics (New York). Wiley-Interscience [John Wiley \& Sons], New York, 2000.

[2] I. Babuška and T. Strouboulis. The finite element method and its reliability. Numerical Mathematics and Scientific Computation. The Clarendon Press Oxford University Press, New York, 2001.

[3] R. Beck, R. Hiptmair, R.H.W. Hoppe, and B. Wohlmuth. Residual based a posteriori error estimators for eddy current computation. M2AN Math. Model. Numer. Anal., 34(1):159-182, 2000.

[4] D. Braess and J. Schöberl. Equilibrated residual error estimator for edge elements. Math. Comp., 77(262):651-672, 2008.

[5] J. Chen, Y. Xu, and J. Zou. Convergence analysis of an adaptive edge element method for Maxwell's equations. Appl. Numer. Math., 59(12):2950-2969, 2009.

[6] Z. Chen, L. Wang, and W. Zheng. An adaptive multilevel method for time-harmonic Maxwell equations with singularities. SIAM J. Sci. Comput., 29(1):118-138 (electronic), 2007.

[7] P. Clément. Approximation by finite element functions using local regularization. Rev. Française Automat. Informat. Recherche Opérationnelle Sér. RAIRO Analyse Numérique, 9(R-2):77-84, 1975.

[8] S. Cochez-Dhondt and S. Nicaise. Robust a posteriori error estimation for the Maxwell equations. Comput. Methods Appl. Mech. Engrg., 196(25-28):2583-2595, 2007. 
[9] M. Costabel, M. Dauge, and S. Nicaise. Singularities of eddy current problems. M2AN Math. Model. Numer. Anal., 37(5):807-831, 2003.

[10] T. Henneron, Y. Le Menach, F. Piriou, O. Moreau, S. Clenet, J.P. Ducreux, and J.C. Verite. Source field computation in ndt applications. IEEE transactions on magnetics, 43(4):1785-1788, 2007.

[11] http://www.compumag.org/jsite/images/stories/TEAM/problem7.pdf.

[12] F. Izsák, D. Harutyunyan, and J.J.W. van der Vegt. Implicit a posteriori error estimates for the Maxwell equations. Math. Comp., 77(263):1355-1386, 2008.

[13] L. Kettunen, K. Forsman, and A. Bossavit. Gauging in Whitney spaces. IEEE transactions on magnetics, 35(3):1466-1469, 1999.

[14] G. Meunier, editor. The finite element method for electromagnetic modeling. ISTE, London, Chap. 3, 2008. Translated from the 2008 French original.

[15] P. Monk. A posteriori error indicators for Maxwell's equations. J. Comput. Appl. Math., 100(2):173-190, 1998.

[16] P. Monk. Finite element methods for Maxwell's equations. Numerical Mathematics and Scientific Computation. Oxford University Press, New York, 2003.

[17] P. Neittaanmäki and S. Repin. Reliable methods for computer simulation, volume 33 of Studies in Mathematics and its Applications. Elsevier Science B.V., Amsterdam, 2004. Error control and a posteriori estimates.

[18] S. Nicaise. On Zienkiewicz-Zhu error estimators for Maxwell's equations. C. R. Math. Acad. Sci. Paris, 340(9):697-702, 2005.

[19] S. Nicaise and E. Creusé. A posteriori error estimation for the heterogeneous Maxwell equations on isotropic and anisotropic meshes. Calcolo, 40(4):249-271, 2003.

[20] Z. Ren. Influence of the r.h.s. on the convergence behaviour of the curl-curl equation. IEEE transactions on magnetics, 32(3):655-658, 1996.

[21] A.A. Rodriguez, R. Hiptmair, and A. Valli. Hybrid formulations of eddy current problems. Numer. Method. Part. Differ. Eq., 21(4):742-763, 2005.

[22] J. Schöberl. A posteriori error estimates for Maxwell equations. Math. Comp., 77(262):633-649, 2008.

[23] Vivette V. Girault and P.A. Raviart. Finite element methods for Navier-Stokes equations, volume 5 of Springer Series in Computational Mathematics. Springer-Verlag, Berlin, 1986. Theory and algorithms.

[24] R. Verfürth. A review of a posteriori error estimation and adaptive mesh-refinement techniques. Chichester and Stuttgart : Wiley and Teubner, Amsterdam, 1996. 